FAST FERROELECTRIC L-BAND TUNER FOR SUPERCONDUCTING CAVITIES

Omega-P, Inc.

291 Whitney Avenue, Suite 401

New Haven, CT 06511

Final Report to US Department of Energy

SBIR Phase I Grant DE-SC000 0922

\title{
FAST FERROELECTRIC L-BAND TUNER FOR SUPERCONDUCTING CAVITIES*
}

\section{CONTENTS}

0. TASKS PROPOSED FOR PHASE I EFFORT p. 2

I. INTRODUCTION 2

II. TECHNICAL APPROACH 3

III. TUNER BASED ON SMALL FERROELECTRIC RING ASSEMBLIES 4

IIIa. General considerations 4

IIIb. Simulation of tuner configuration employing small ferroelectric ring assemblies $\quad 5$

IIIc. Theoretical relation between phase shift and losses

IIId. Experimental tests of ferroelectric assemblies 20

$\begin{array}{ll}\text { IIIe. Power handling considerations } & 24\end{array}$

$\begin{array}{ll}\text { IV. CONCLUSIONS } & 25\end{array}$

$\begin{array}{ll}\text { REFERENCES } & 29\end{array}$

\section{SUMMARY}

Design, analysis, and low-power tests are described on a ferroelectric tuner concept that could be used for controlling external coupling to RF cavities for the superconducting Energy Recovery Linac (ERL) in the electron cooler of the Relativistic Heavy Ion Collider (RHIC) at Brookhaven National Laboratory (BNL). The tuner configuration utilizes several small donut-shaped ferroelectric assemblies, which allow the design to be simpler and more flexible, as compared to previous designs. Design parameters for 704 and $1300 \mathrm{MHz}$ versions of the tuner are given. Simulation results point to efficient performance that could reduce by a factor-of-ten the RF power levels required for driving superconducting cavities in the BNL ERL.

*Supported by DoE Office of Nuclear Physics.

Work on this project was carried out by senior research scientist Dr. S.Yu. Kazakov, with important input from consultant Dr. V.P. Yakovlev of FNAL and research scientist Dr. S.V. Shchelkunov of Yale University, with Dr. J.L. Hirshfield serving as Principal Investigator.

The report was written by Dr. Hirshfield.

December 12, 2011, revised July 3, 2012 


\section{Omega-P, Inc.}

Final Report on Phase I DoE SBIR grant DE-SC000 0922

\section{FAST FERROELECTRIC L-BAND TUNER FOR SUPERCONDUCTING CAVITIES}

\section{TASKS PROPOSED FOR PHASE I EFFORT}

At the writing of the Phase I proposal in Fall 2008, the following tasks were proposed:

Task a: Further optimize the design for a full-scale triple-decker $700 \mathrm{MHz}$ tuner.

Task b: Carry out simulation studies of the radial line reflecting tuner, with the aim of comparing its performance with the planar triple-decker design so as to select the superior design for full development during Phase II.

Task c: If results of Task b are sufficiently encouraging, build a $1300 \mathrm{MHz}$ prototype for lowpower cold tests, and carry out the tests to confirm the predictions.

Task d: Coordinate with Euclid Techlabs LLC to improve the recipe for ferroelectric material with the aim of lowering its loss tangent without undue sacrifice in tunability, and to develop a technique for reliable low-temperature soldering or brazing to copper plates of metalized ferroelectric and ceramic bars. Attention will be directed towards developing a technique for the braze fillet to cover triple-point corners, so as to guard against field-enhanced breakdown at those joints. Test both improvements at $1300 \mathrm{MHz}$ in one-third scaled three-deck structure, or in radial line tuner.

Task $\boldsymbol{e}$ : Develop or acquire an improved fast high-voltage pulser and/or pulser circuit for rapid phase shifting. Test one or both tuner at low power to determine their shortest response time.

Task f: Assemble Phase I results and confer with collaborators to plan and compose a Phase II project and proposal.

Task g: Write a final report, and as work progresses deliver oral presentations at relevant conferences and workshops, and at sponsor's reviews as requested. Publish significant results in refereed journals.

\section{INTRODUCTION}

Under Topic 46a in the DoE 2009 SBIR Program Solicitation, Phase I proposals were sought to improve or advance superconducting and room-temperature materials or components for RF devices used in particle accelerators. Areas of interest included "...fast ferroelectric microwave components that control reactive power for fast tuning of cavities or fast control of input power coupling." Omega-P, Inc. submitted a proposal in response, entitled "Fast Ferroelectric L-Band Tuner For Superconducting Cavities," and was awarded the Phase I grant DE-SC000 0922 with the same title. The overall goal of the Phase I R\&D program was to design, build, and test a $700 \mathrm{MHz}$ fast electrically-driven ferroelectric tuner for controlling the external coupling to RF cavities for the superconducting Energy Recovery Linac (ERL) in the electron cooler of the Relativistic Heavy Ion Collider (RHIC) at Brookhave National Laboratory. The Phase I objectives were met, as will be described in detail in Section II. However, the proposal submitted for continuation of the work under a Phase II grant was not approved, despite 


\section{FAST FERROELECTRIC L-BAND TUNER FOR SUPERCONDUCTING CAVITIES}

compelling evidence that a tuner design was sufficiently perfected to allow engineering, construction, and testing to be undertaken with confidence.

\section{TECHNICAL APPROACH}

In ERLs, there are several factors which significantly affect the amount of power required from the wall-plug to provide the RF-power level necessary for operation. When beam loading is small, the power requirements are determined by $(i)$ ohmic losses in walls, (ii) imbalance between the beam currents, and (iii) microphonics. These factors typically require a substantial change in the coupling between the cavity and feeding line, which results in a growth in the necessary bandwidth, and demands a significant amount of additional power. If beam loading is not small, there is a variety of beam-drive phase instabilities to be managed, and microphonics will still remain an issue, so there remain requirements for additional power. Moreover ERL performance is sensitive to changes in beam arrival time, since any such change is equivalent to phase instability with its vigorous demands for additional power.

It is presently recognized that the aforementioned effects can be compensated by several means, which seek either to actively change the cavity dimension in such a way that the detuning caused by phase instabilities and/or microphonics is nearly-perfectly offset $[1,2]$, or to apply a corrective phase shift to the RF wave reflected from the cavity, that later is reintroduced to the cavity to cancel phase instabilities $[3,4]$. Because of statistical nature of phase instabilities and microphonics, it is imperative to employ a negative feedback loop in each approach.

The first line of defense in this strategy is accomplished by internal or external mechanical actuators, or by fast internal mechanical piezoelectric tuners. The second approach utilizes fast ferrite or ferroelectric external-to-cryomodule phase shifters that have the necessary RF-coupling with the cavities. Piezoelectric (and other mechanical) tuners require operation at cryogenic temperatures and thus permit only limited access inside the cryomodule in the event of a failure. Further, the piezoelectric devices have their own mechanical resonances which may interfere with control system performance if their self-resonance frequency overlaps with the microphonic excitation to be controlled [5]. It is also questionable as to whether the piezo-tuners are efficient enough to counter the phase instabilities at high frequencies. Ferrite phase shifters $[6,7,8]$, in particular one that is being developed at CERN for the Superconducting Proton Linac [4], are presently limited in their response times to $\sim 30 \mathrm{~ms}$, whereas the response time is required to be no larger than $\sim 1 \mathrm{~ms}$. The limitation comes mainly from eddy currents developed within the ferrite itself [7]. The response time requirements $(<1 \mathrm{~ms})$ are dictated by the necessary phase and amplitude stabilities of about $0.06^{\circ}$ and $3 \times 10^{-4}$, respectively. The cited numbers are for the Cornell ERL [9], but the ERL for the electron cooler project at BNL [10] has requirements of the same order-of-magnitude. Gain in the control feedback loop should be high enough, and its bandwidth wide enough, to insure this high degree of stability. This translates to a bandwidth of about $1 \mathrm{MHz}$, and rules out contemporary ferrite tuners with their narrow bandwidths. 


\section{Omega-P, Inc.}

Final Report on Phase I DoE SBIR grant DE-SC000 0922

\section{FAST FERROELECTRIC L-BAND TUNER FOR SUPERCONDUCTING CAVITIES}

Omega-P initially described in its Phase I proposal two alternative concepts for a fast electrically-controlled ferroelectric $700 \mathrm{MHz}, 50 \mathrm{~kW}$ phase shifter for ERL applications.* These concepts are the triple-decker waveguide tuner, and the radial line reflecting tuner. One objective during Phase I was to compare the predicted performance of these two approaches, so as to select the superior design for subsequent full development. However, during Phase I, issues with each of these arose and a new attractive concept was invented, namely a multi-ring concept. This new concept is based on the utilization of several small donut-shaped ferroelectric assemblies, which allow the design to be simpler and more flexible, as compared to the previous designs. Each assembly consists of a metalized ferroelectric ring brazed between two copper cups. The assembly allows separate tuning and testing of each assembly prior to installation. The assembly was to have been developed by collaborating firm Euclid Techlabs LLC within the scope of its own DoE-sponsored grants.

In this report, we describe the new multi-ring tuner, with specifications suitable for the $700 \mathrm{MHz}$ ERL application. The device will allow changing the RF-coupling during the cavity filling process in order to effect significant RF power savings, and also will provide rapid compensation for beam imbalance and allow for fast stabilization against phase fluctuations caused by microphonics, beam-driven instabilities, etc. The tuner is predicted to allow a reduction of about ten times in the required power from the RF source, as compared to a compensation system with narrower bandwidth. During Phase II, Omega-P proposed to build a $700 \mathrm{MHz}$ version of the tuner, to check its underlying principles, and to make high-power tests at power densities aimed towards controlling $50 \mathrm{~kW}$ of average power. Steps towards this goal would be limited by, among other factors, losses in the actual ferroelectric elements in the ferroelectric assemblies. As Euclid, during anticipated progress under its Phase II development program, was to reduce the ferroelectric material loss tangent and the concomitant power loss in its ferroelectric assemblies, the average power-handling capability of the Omega-P tuner would rise. It was thus anticipated that the Phase II development of the $700 \mathrm{MHz}$ tuner would be iterative, but the pace and ultimate power-handling level of the tuner was difficult to predict at such an early stage in Euclid's development program. But, as mentioned, these developments did not materialize since further grant support was unavailable.

\section{TUNER BASED ON SMALL FERROELECTRIC RING ASSEMBLIES}

\section{IIIa. General considerations.}

The ultimate goal of this project was to develop a $704 \mathrm{MHz}$ fast tuner for the superconducting cavities in ERL that are part of the electron cooler for RHIC. Key objectives were both design and technological. The technical objective of the Phase I program was to develop a preliminary design of a fast external tuner for coupling of ERL cavities using electrically-

\footnotetext{
*The actual frequency for the BNL ERL is $704 \mathrm{MHz}$; we will build prototypes accordingly.
} 


\section{Omega-P, Inc.}

Final Report on Phase I DoE SBIR grant DE-SC000 0922

\section{FAST FERROELECTRIC L-BAND TUNER FOR SUPERCONDUCTING CAVITIES}

controlled ferroelectric phase shifters. Each tuner should allow rapid changes in the coupling of an ERL cavity with the feed line at an average power level of up to $50 \mathrm{~kW}$ at $704 \mathrm{MHz}$. The tuner design must involve detailed analytical studies and numerical simulations of a phase shifter in order to minimize electric fields and power losses in the ferroelectric elements, providing at the same time the required range and speed of RF phase changes.

During Phase I, the two concepts for the phase shifter were initially considered:

a) a triple-decker concept, similar to one developed for ILC applications [11], and

b) a radial line reflecting tuner.

However, experience with the triple-decker concept demonstrated problems with highvoltage breakdown and brazing of ceramics to copper walls. In addition, the concept required two types of ceramic elements: ferroelectric bars and linear ceramic matching bars. The radial line reflecting phase shifter requires a ferroelectric ring of substantial physical size that appeared to be difficult to sinter, metalize and braze. Thus, after detailed analysis of electrodynamic and technological problems of both concepts, these were downgraded in priority in favor of a new concept based on use of a number of small ferroelectric ring assemblies. These assemblies can be standardized, and are of relatively small size that should be relatively easy to manufacture, individually tuned, cooled, and tested. Furthermore, this new phase shifter design requires no linear ceramic matching elements.

The ferroelectric assemblies were to be developed and fabricated to Omega-P specifications for the $704 \mathrm{MHz}$ tuner by Euclid TechLabs LLC, with support under their own anticipated Phase II SBIR grant from DoE that concentrates on development of ferroelectric ceramics and of technological means to incorporate these into brazed assemblies. Clearly, success with Omega-P's tuner would depend upon Euclid's success in its development of the ferroelectric material, particularly for material having a dielectric constant reduced from 500-600 to about 100 , with a tunability of about $6 \%$ at an electric field of $15 \mathrm{kV} / \mathrm{cm}$, and with a loss tangent of $5 \times 10^{-4}$. Fabrication by Euclid of the certified assemblies would also be essential, so as to insure that contamination of the ceramic that could arise unintentionally during brazing by others, who may not have access to Euclid's proprietary processes, will not have altered the critical characteristics of the ferroelectric material itself.

\section{IIIb. Simulation of tuner configuration employing small ferroelectric ring assemblies.}

A tuner may be designed as a combination of two fully-reflecting phase shifters and a 3dB hybrid, as shown in Fig. 1. This device will in principle provide any desired degree of reflection and any desired phase, independently of one another. Thus, the problem is reduced to the design of a fully-reflecting fast-response, low-loss phase shifter. 


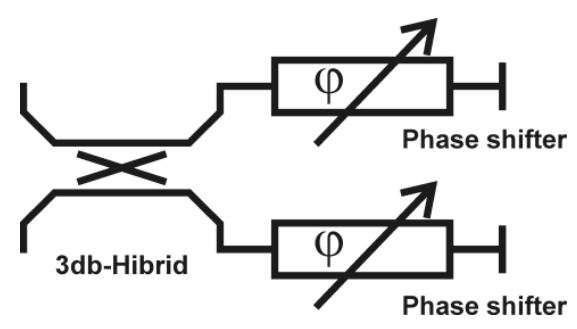

Fig. 1. Combination of 3-dB hybrid and two shorted phase shifters amounts to a tuner.

The proposed overall device configuration is shown in Fig. 2 (see, for example, [8]) that can allow fast electrically-controlled coupling and reflecting phase changes. This device is based on a magic- $T$ with two coaxial phase shifters containing ferroelectric assemblies. Changing the phase shifts of the reflected signal from $0^{\circ}$ to $90^{\circ}$ will change the transmission coefficient from 0 to 1 , or the phase from $0^{\circ}$ to $90^{\circ}$, independently of one another [16]. For a symmetric magic-T, the power level at each phase shifter is half of the total power incident in the input port, which thereby reduces the temperature rise in the phase shifter's active element (ferroelectric) that must be accounted for in the design. The phase shifter itself was originally proposed to be designed either as a triple-decker waveguide concept, similar to one considered for ILC applications [11], or a radial line reflecting tuner. Applying bias voltage across the ferroelectric assemblies effects a change in dielectric permittivity of the ferroelectric, which causes a phase advance of the RF wave in the phase shifter, and thus a change in coupling between the cavity and the RF source. 


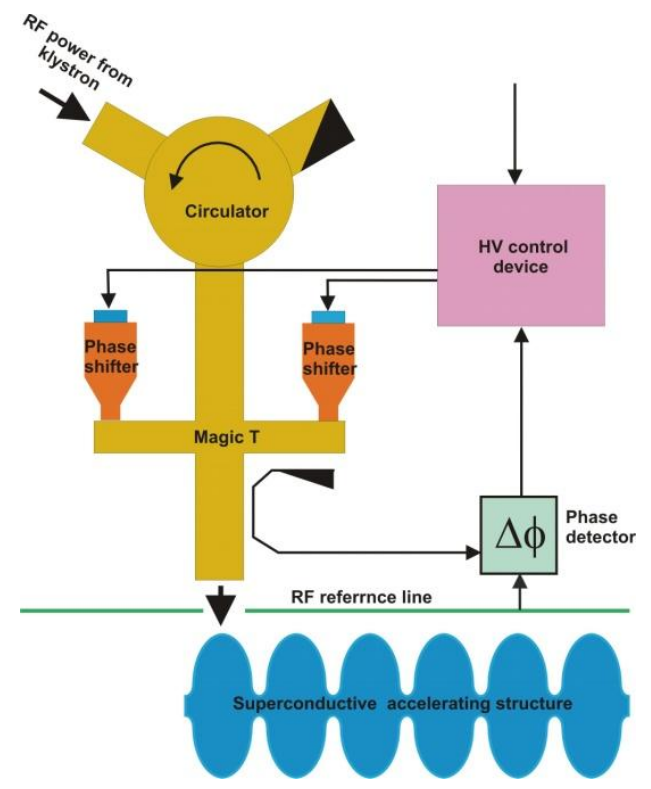

Fig. 2. A possible configuration for SRF cavity phase and amplitude control Using two external tuners (reflecting phase shifters) and a magic-T. Also shown is a schematic of the feedback loop that would be employed for rapid automated stabilization.

The earlier triple-decker waveguide concept and the radial line concept for the phase shifter both exhibit serious problems with high-voltage breakdown at ceramic-metal boundaries, and brazing of ceramics to the copper walls. In addition, the triple-decker concept including two types of the ceramic elements: ferroelectric bars and matching bars made of the linear ceramics. The radial line reflecting tuner requires a large ferroelectric ring, which appears to be difficult to sinter, metalize and braze. After detailed analysis of electrodynamics and technological problems of both concepts, a new concept was invented based on the use of a number of small ferroelectric rings. The new concept utilizes standard simple ferroelectric assemblies of relatively small size that may be easily manufactured, tuned and tested separately.

In the new concept, one or more ferroelectric assemblies behave as cavities connected as transmission components within waveguides, as shown in Fig. 3. Changing the resonant frequency of each cavity by voltage-tuning the ferroelectric assemblies can change the phase of the transmitted signal while keeping the SWR within an acceptable low range. Further, in the new concept the ferroelectric assembly is placed inside the transmission line, and the RF energy partially flows past the assembly, as shown in Fig. 4. The coupling is adjusted not by a dielectric matching element, but by selection of the relative sizes of the transmission line and the ferroelectric assembly. An example of such a cavity is shown in Fig. 5, where the ferroelectric assembly is placed into a waveguide. Transmission and reflection characteristics are shown in Fig 5d. One can see that by changing the resonant frequency of the ferroelectric ring (i.e., 
dielectric constant of the ferroelectric material) it is possible to change the phase of the transmitted wave.
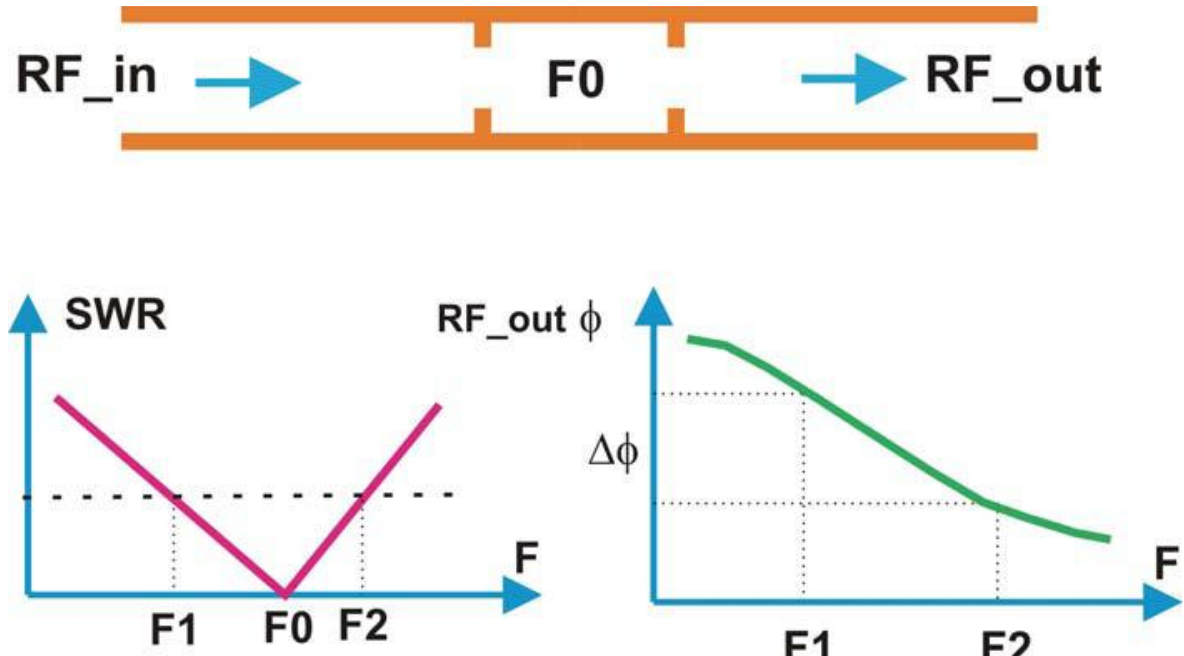

Fig. 3. Equivalent circuit of the strongly coupled ferroelectric phase shifter.

The ferroelectric element works as a transmission-type cavity.

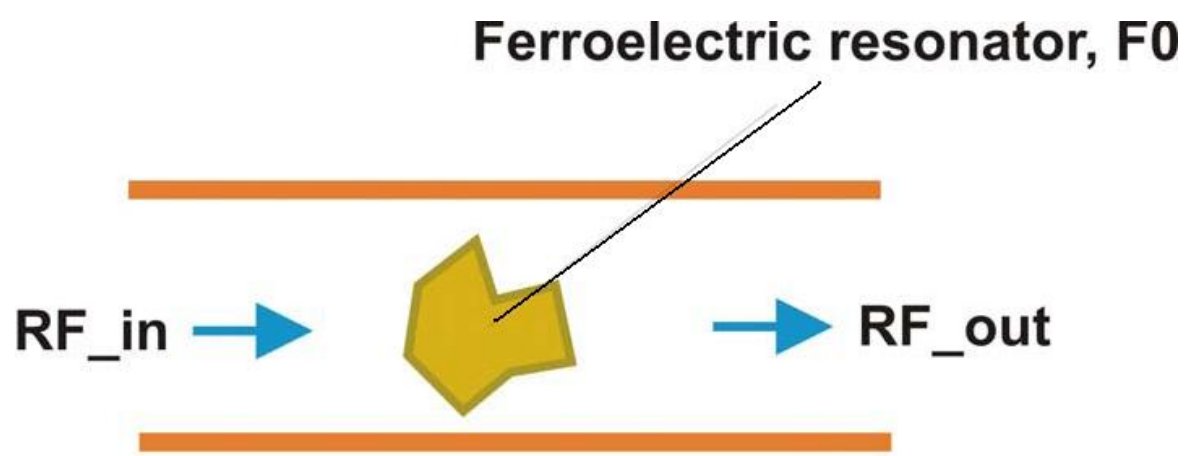

Fig. 4. Sketch of the new concept of the phase shifter.

The simplest and technologically-convenient shape for the ferroelectric element is a pillbox. In Fig. 6 are shown several modes in a ferroelectric pillbox. The desired mode of operation is $\mathrm{TM}_{020}$, because it has maximum coupling to the waveguide field. But the pillbox has a parasitic mode $\mathrm{TM}_{012}$ close to the operating mode, as also seen in Fig. 6 . For these examples, the dielectric constant $\varepsilon=300$, and the pillbox radius is $8.34 \mathrm{~mm}$. Examples shown are for a $1300 \mathrm{MHz}$ tuner, but scaling these to $704 \mathrm{MHz}$ is straightforward. 


\section{FAST FERROELECTRIC L-BAND TUNER FOR SUPERCONDUCTING CAVITIES}

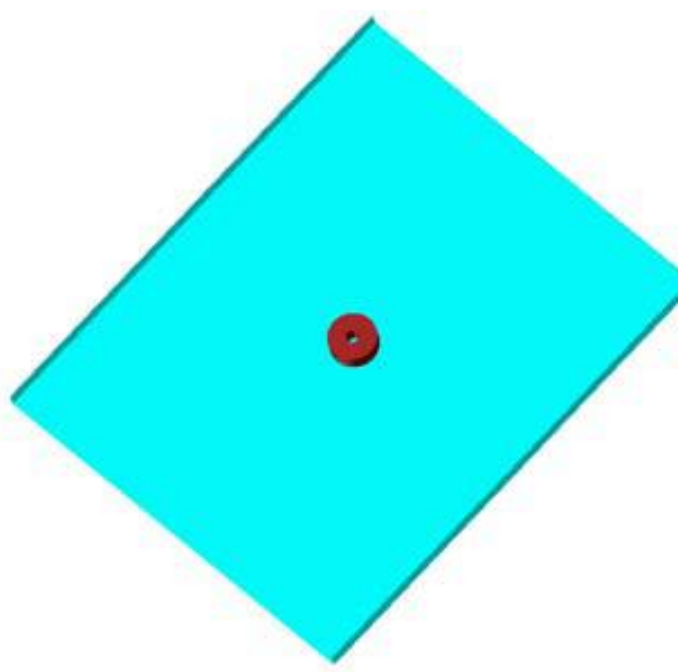

(a)

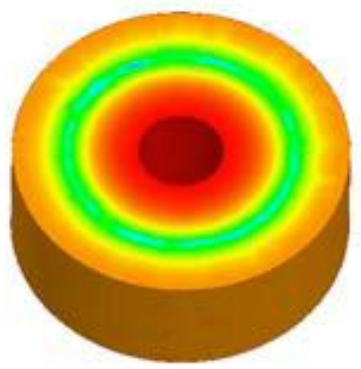

(b)

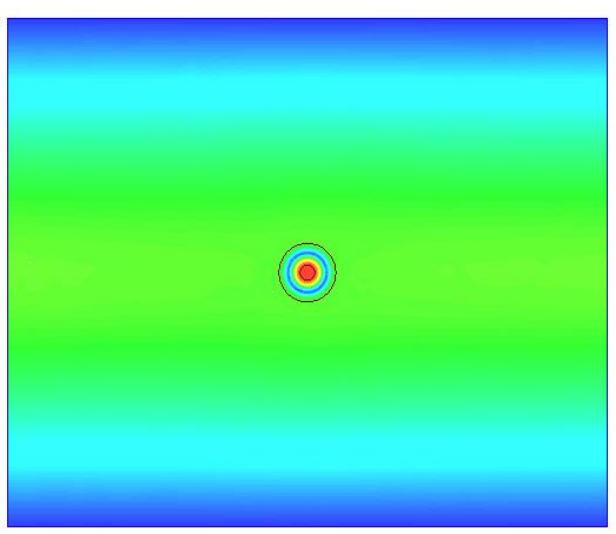

(c)

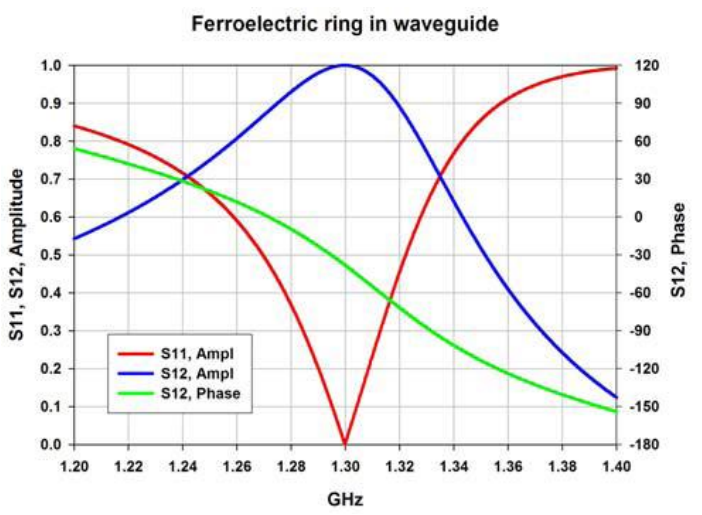

(d)

Fig. 5. (a) Ferroelectric ring inside a waveguide; (b) field distribution in ferroelectric ring; (c) field in the waveguide; (d) transmission and reflection of the ferroelectric ring inside waveguide.

Note that this example is for a $1300 \mathrm{MHz}$ tuner; scaling to $700 \mathrm{MHz}$ is straightforward.

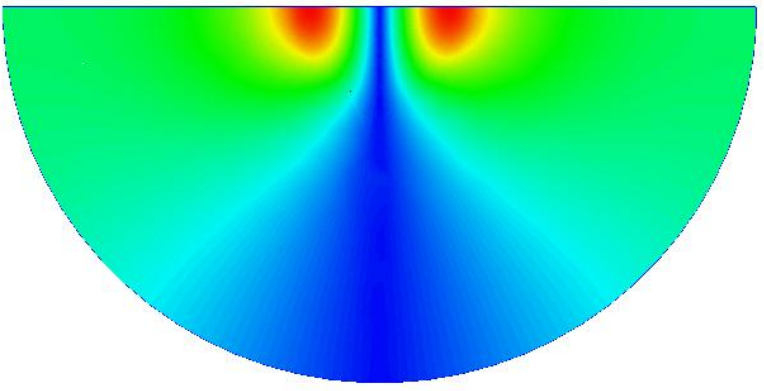

$\mathrm{TM}_{011}, \mathrm{f}=780 \mathrm{MHz}$

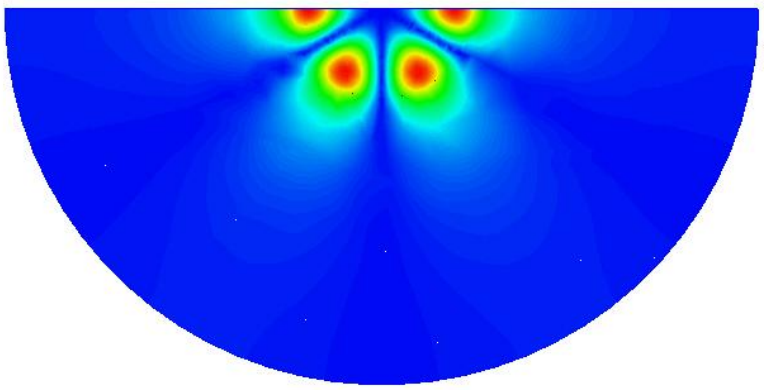

$\mathrm{TM}_{013}, \mathrm{f}=1695 \mathrm{MHz}$ 
FAST FERROELECTRIC L-BAND TUNER FOR SUPERCONDUCTING CAVITIES
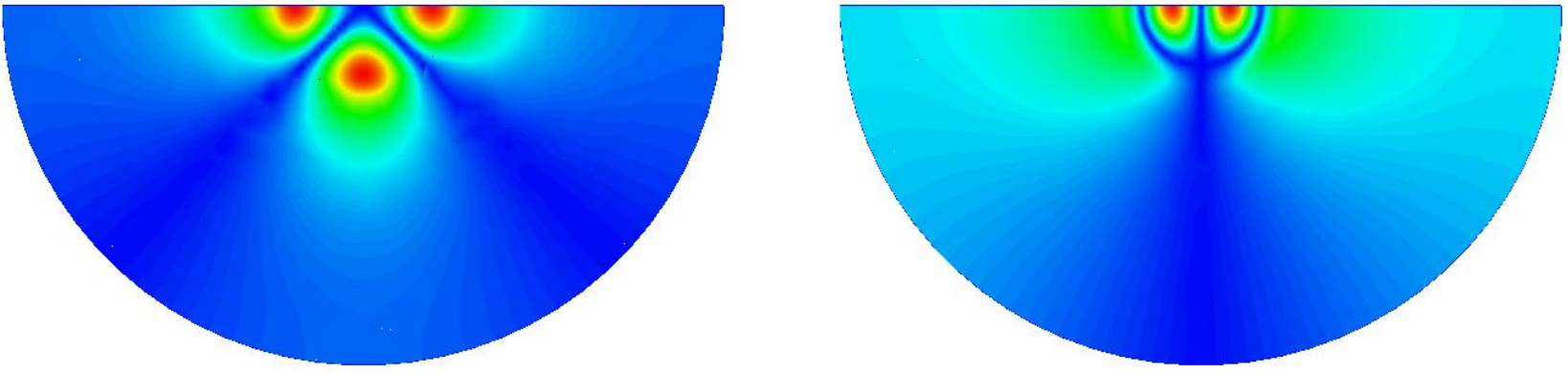

$\mathrm{TM}_{012}, \mathrm{f}=1263 \mathrm{MHz}$
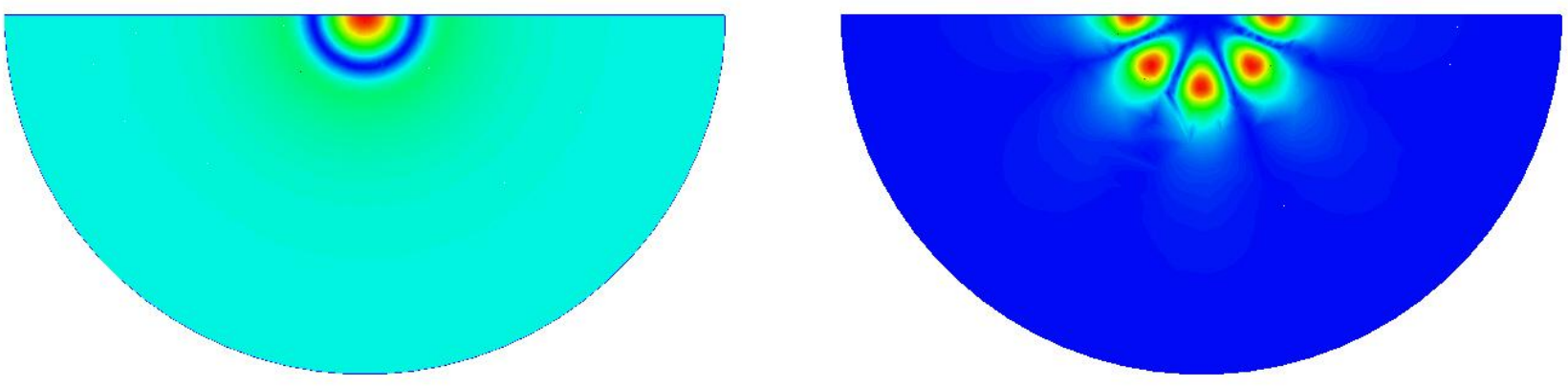

$\mathrm{TM}_{020}, \mathrm{f}=1300 \mathrm{MHz}$

$\mathrm{TM}_{022}, \mathrm{f}=1818 \mathrm{MHz}$

$\mathrm{TM}_{014}, \mathrm{f}=2106 \mathrm{MHz}$

Fig. 6. Modes in a ferroelectric pillbox.

In order to avoid problems with the nearby mode $\mathrm{TM}_{012}$ mode, it is possible to use a ring instead of a pillbox. A reference diagram of the ring is shown in Fig. 7. In Fig. 8 the frequencies of the lowest modes of a cylindrical coaxial ring are shown versus its internal radius. The external radius is $8.34 \mathrm{~mm}$, and the dielectric constant $\varepsilon=290$. One can see that the internal radius should be in the range of 2-3 $\mathrm{mm}$. If one keeps the frequency of the operating $\mathrm{TM}_{020}$ mode to be $1.3 \mathrm{GHz}$, the external radius should be $9.53 \mathrm{~mm}$. and internal radius is should be 2.5 $\mathrm{mm}$, so the operating $\mathrm{TM}_{020}$ mode is mid-way between the parasitic modes, $\mathrm{TM}_{210}$ and $\mathrm{TM}_{310}$. 


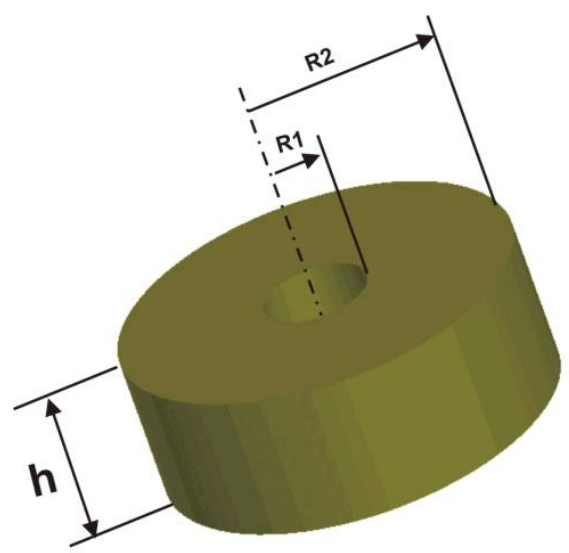

Fig. 7. Reference geometry for a ferroelectric ring, showing dimensional quantities.

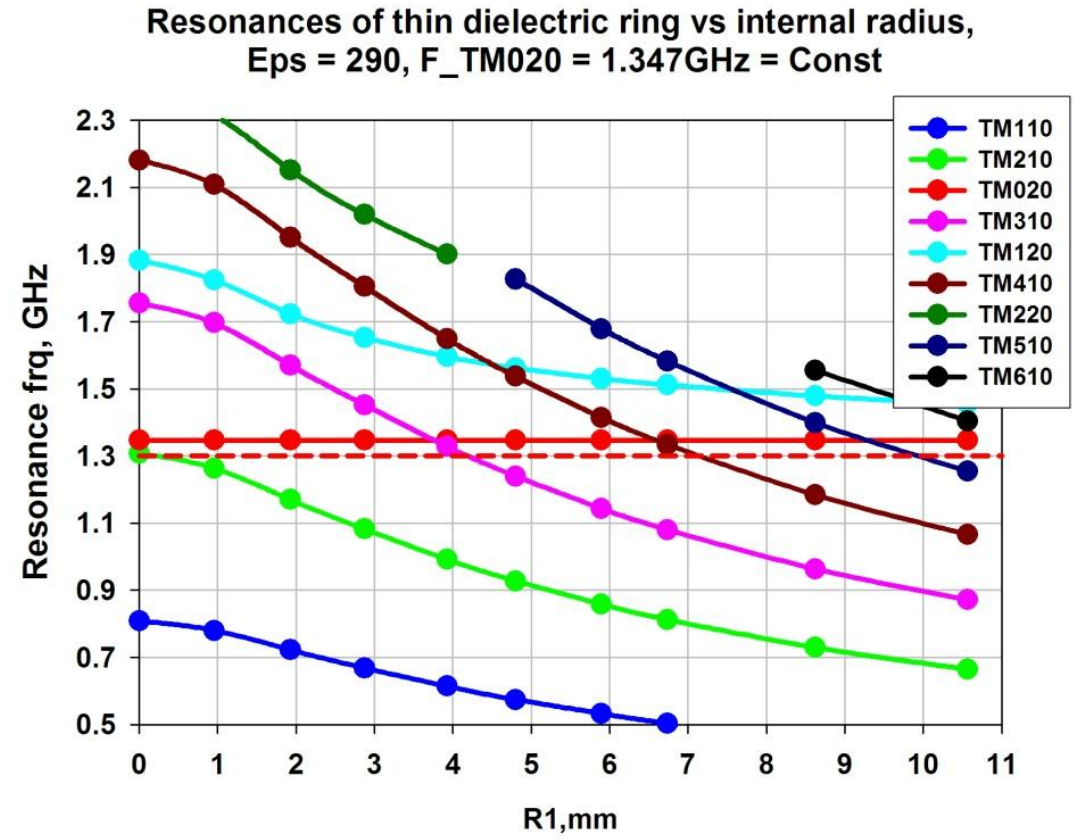

Fig. 8. Frequencies of the lowest modes in a ferroelectric ring versus the ring internal radius.

Fig. 9 shows the dependence of the external radius $R_{2}$ of the ring on the internal radius $R_{1}$ of center hole. The frequency of operating mode $\mathrm{TM}_{020}$ is kept constant at $1.347 \mathrm{GHz}$. 


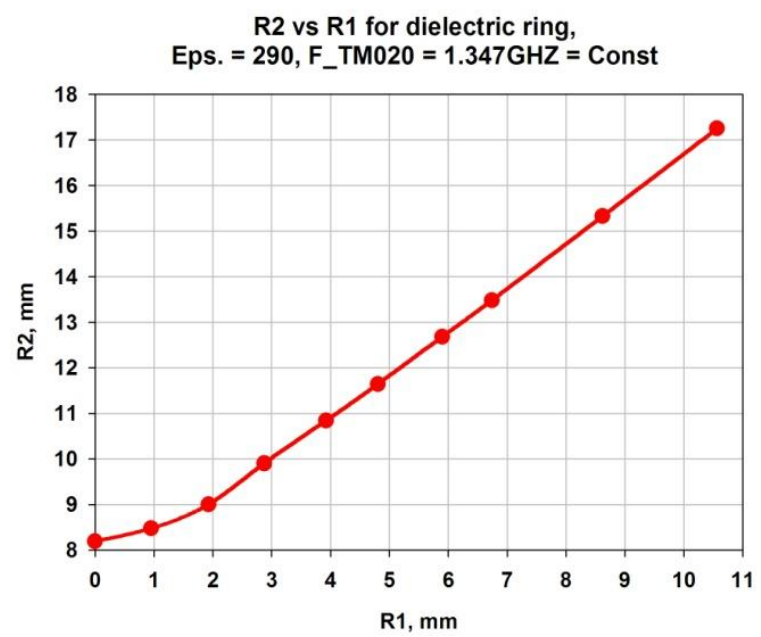

Fig. 9. Ring external radius $R_{2}$ versus the internal radius $R_{1}$ for the fixed frequency of the operating mode, namely $1.3 \mathrm{GHz}$. Dielectric constant $\varepsilon=300$.

In Fig. 10 is shown the dependence of the three neighboring parasitic modes versus the ring height $h$. The values of $R_{2}$ and $R_{1}$ are fixed at optimam, $9.53 \mathrm{~mm}$ and $2.5 \mathrm{~mm}$ respectively.

Parasitic resonances vs height of tablet.

R1 and R2 are optimal.

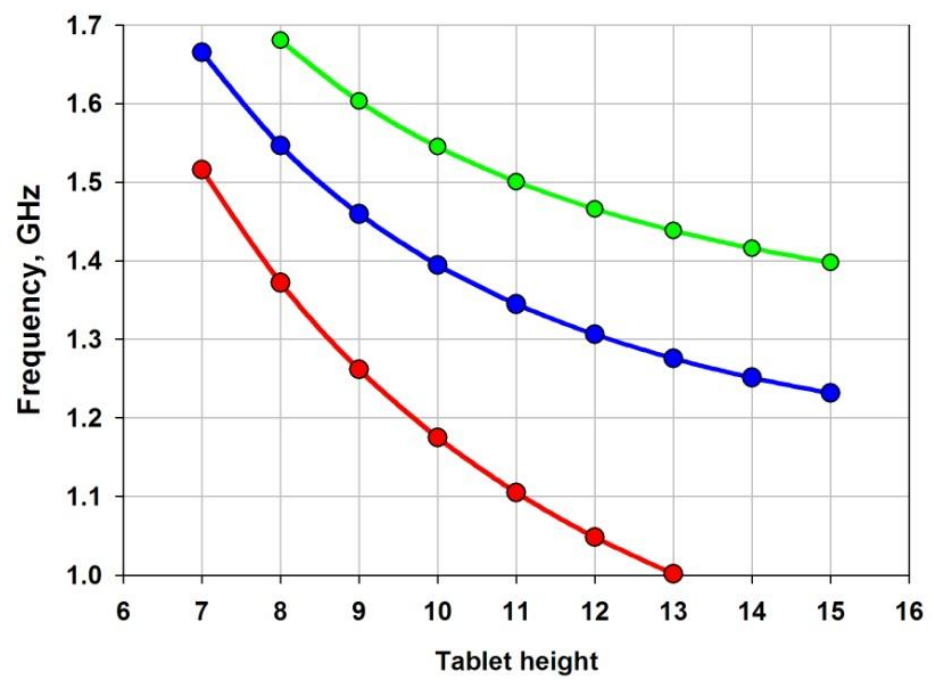

Fig. 10. Parasitic mode frequencies versus the ring height.

The ferroelectric assembly comprises the ferroelectric ring brazed between two metal cups, as depicted in Fig. 11. Edges of the cups are rounded in order to have improved breakdown strength. The small rounded sitting places necessary to shield triple points near the ferroelectric edges, are not shown in the figure. 


\section{FAST FERROELECTRIC L-BAND TUNER FOR SUPERCONDUCTING CAVITIES}
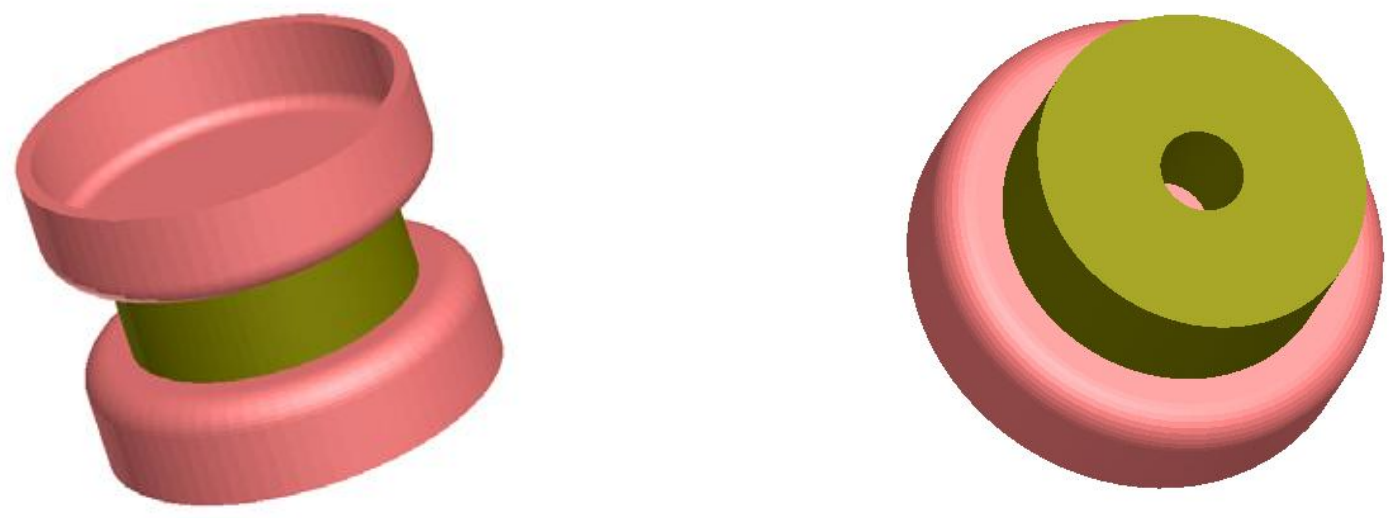

Fig.11. Concept of the ferroelectric assembly.

The ferroelectric assembly positioned in a waveguide is shown in Fig. 12. The edges of the waveguide near the sitting hole in the waveguide walls are rounded as well.

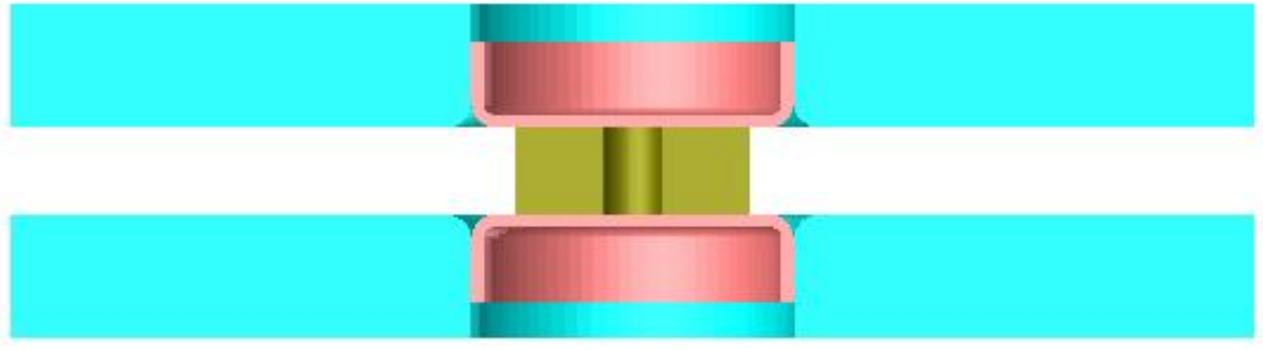

Fig. 12. A ferroelectric assembly inside a waveguide.

To widen the passband of a phase shifter and to make its tunability higher, one can use several rings in line. Some results of simulation are presented below in Figs 13-14 to demonstrate this. Note that one can change strength of electric field inside the ferroelectric rings by shifting the assemblies away from center of waveguide.

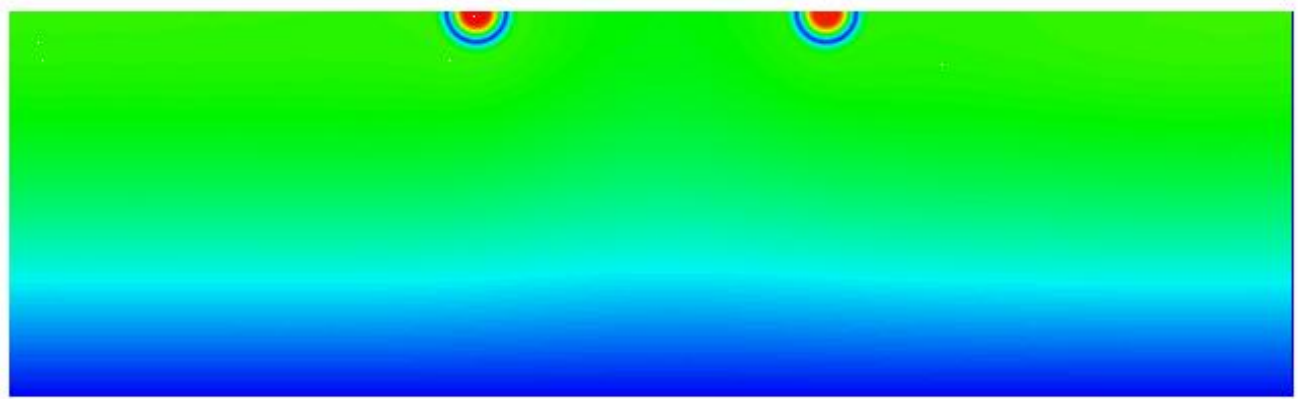

Fig. 13. Fields within a waveguide with the two ferroelectric rings. 
Two ring in the center of waveguide, $\mathrm{R} 1=\mathbf{2} .4 \mathrm{~mm}, \mathrm{R} 2=9.46 \mathrm{~mm}$

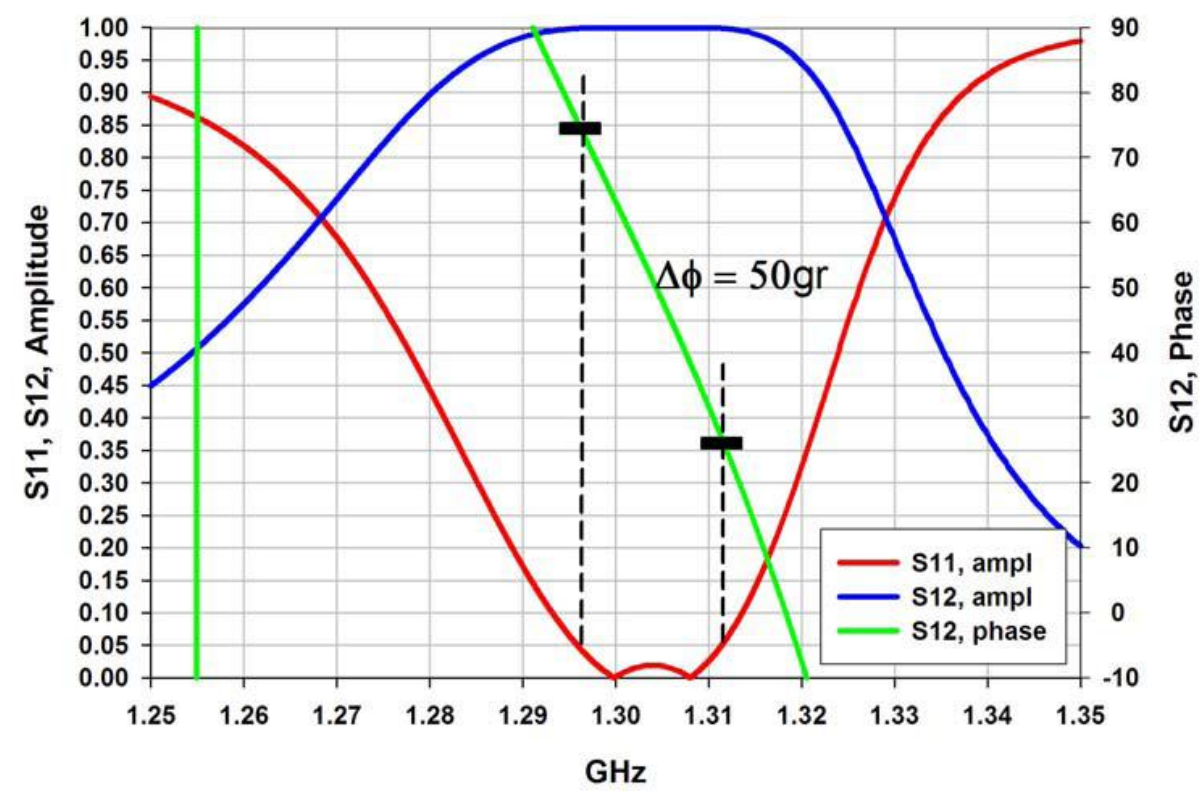

Fig 14. Transmission characteristic of a waveguide loaded with two rings assemblies. The phase shift is $\sim 50 \mathrm{deg}$ in the range where $S_{11}$ is not higher than 0.05.

One design concept for a multi-ring structure is a quasi-rectangular coaxial line loaded by four ring elements, as shown in Fig. 15. The E-fields in this structure are shown in Fig. 16.

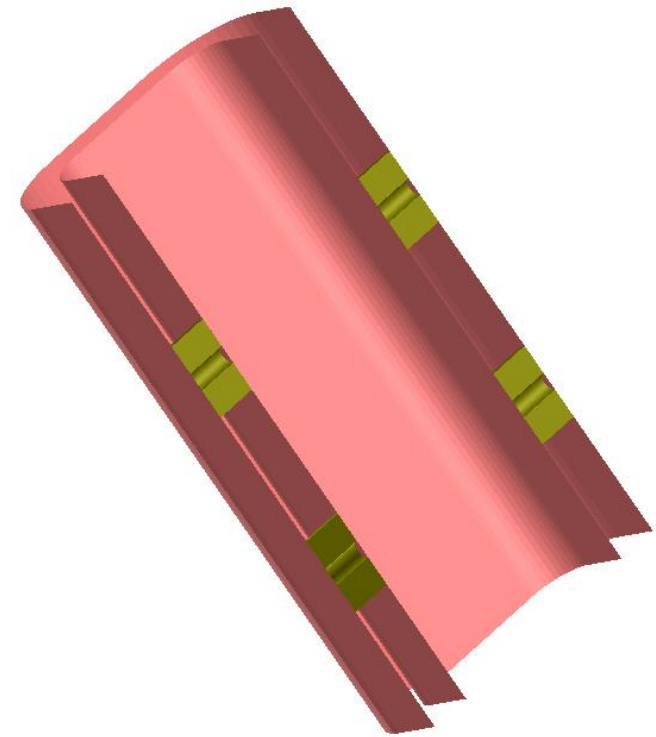

Fig. 15. Geometry of the rectangular coaxial line loaded by four ferroelectric assemblies.

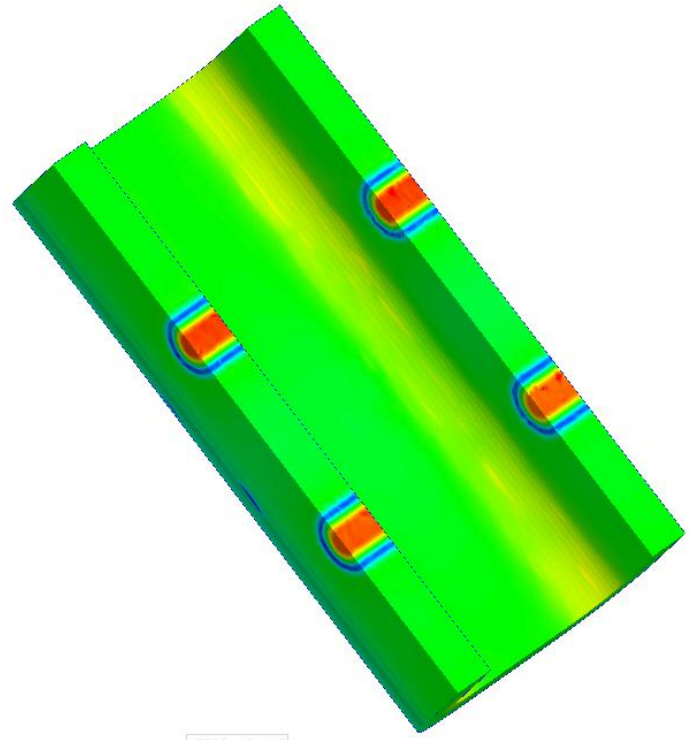

Fig. 16. E-fields in the quasi-rectangular coaxial line loaded by four ferroelectric assemblies. 
Transmission characteristics for this structure with different dielectric constants are shown in Fig. 17.

\section{Ferroelectric disks in coaxial line}

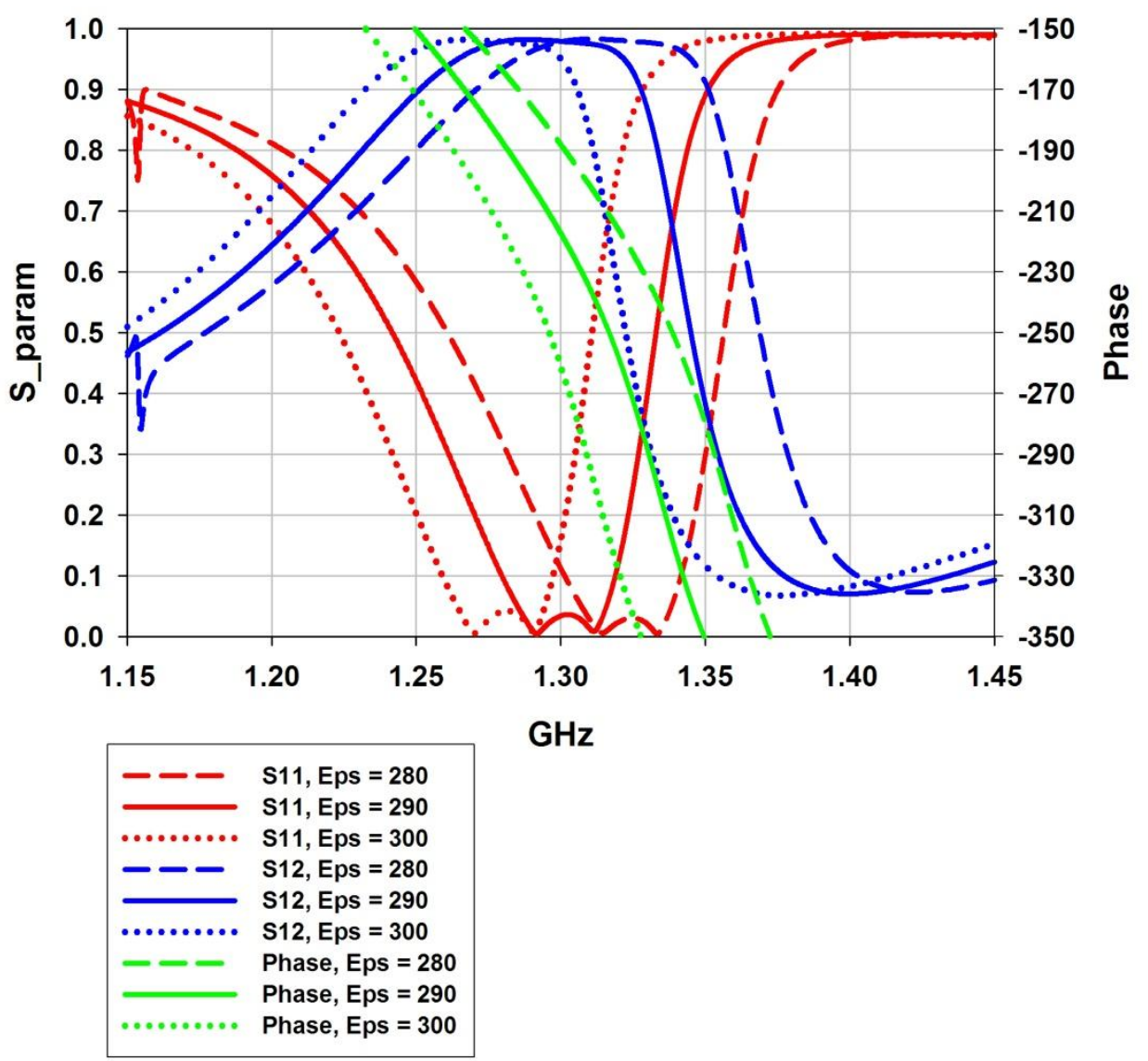

Fig. 17. Transmission characteristics for different dielectric constants.

In Fig.18. The losses are shown for the different dielectric constants for a loss tangent of $1 \times 10^{-3}$. Losses in the copper walls are taken into account in this calculation. 

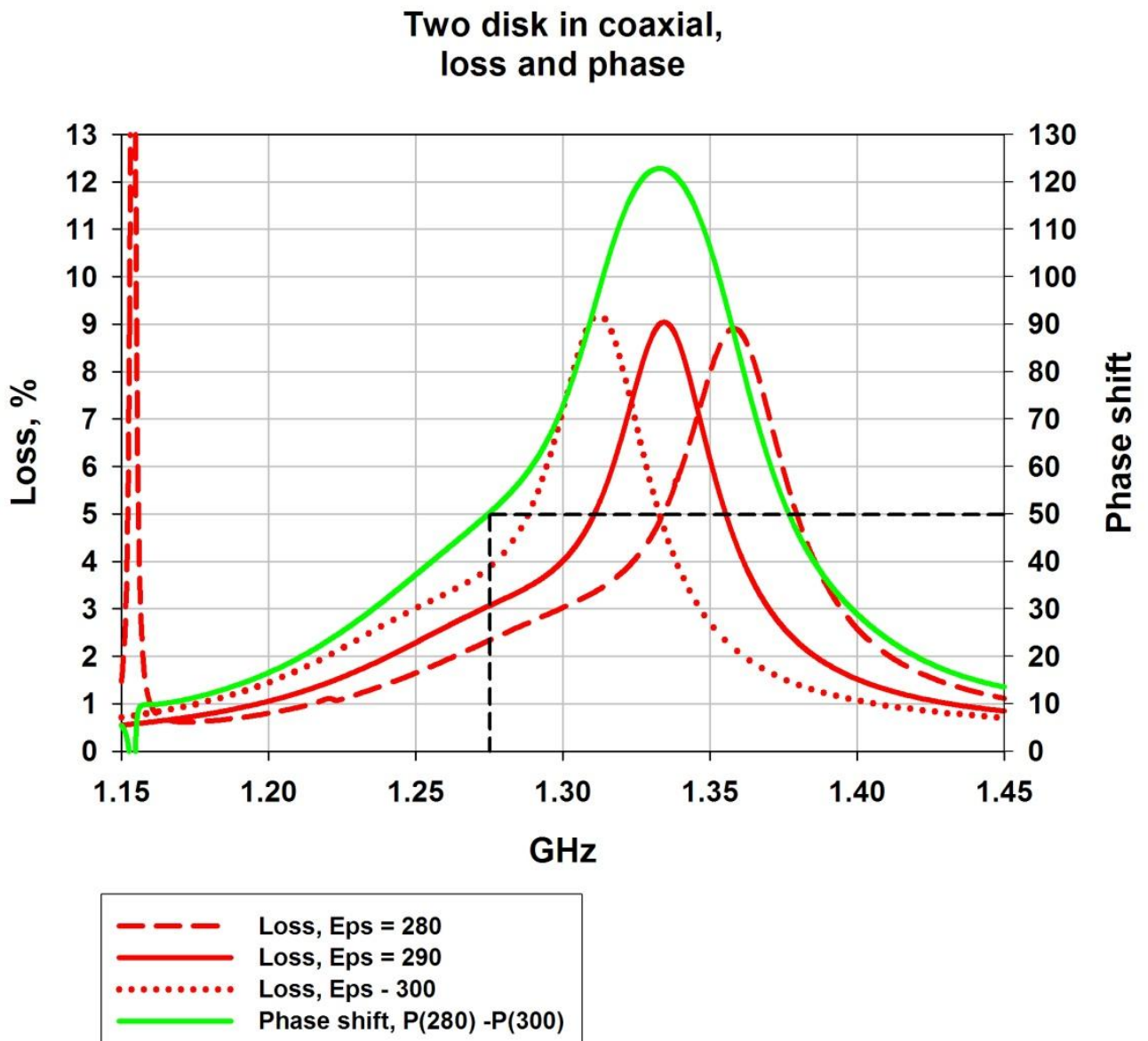

Fig.18. Phase shift and losses are shown for different dielectric constants for a loss tangent of $1 \times 10^{-3}$.

For a phase shift of $50^{\circ}$ the losses are $3 \%$. If the line is shorted, the phase of the reflected wave will change by $100^{\circ}$, and losses will be $6 \%$. Typically, for ferroelectrics, the loss tangent is proportional to frequency, and at $704 \mathrm{MHz}$ for this material one can expect a loss tangent of about $5 \times 10^{-4}$, and losses for the reflected wave of about $3 \%$. Note that with two phase shifters and a total incident power of $50 \mathrm{~kW}$, the loss in each structure would be about $750 \mathrm{~W}$. This clearly indicates that a multiplicity of structures would be probably be required, or that the loss tangent of the ferroelectric material should be reduced if possible, or both, for controlling a power level of $50 \mathrm{~kW}$. For lower power, the challenge is clearly reduced proportionally.

The losses in the ferroelectric may be estimated from the equation below, as derived in Section IIc.

$$
\frac{P_{\text {loss }}}{P_{0}} \approx 4 \operatorname{tg} \delta \frac{\phi_{0} \varepsilon}{\Delta \varepsilon} .
$$




\section{FAST FERROELECTRIC L-BAND TUNER FOR SUPERCONDUCTING CAVITIES}

where symbols in this equation are given by:the incident power $P_{0}$, the maximum possible change of the dielectric constant of the ferroelectric $\Delta \varepsilon$, and the desired change of the phase of the reflected signal $2 \phi_{0}$. For $\phi_{0}=50^{\circ}$, a loss tangent of $5.4 \times 10^{-4}$, and a relative change of the dielectric constant of $6.7 \%$, this formula gives losses of $2.8 \%$, a value that is very close to that found in the simulations (3\%).

A design concept for the phase shifter together with a matching taper is shown in Fig. 19. In order to sustain a high average power, the phase shifter may contain a number of coaxial structures, as depicted in Fig. 20 where four are shown.

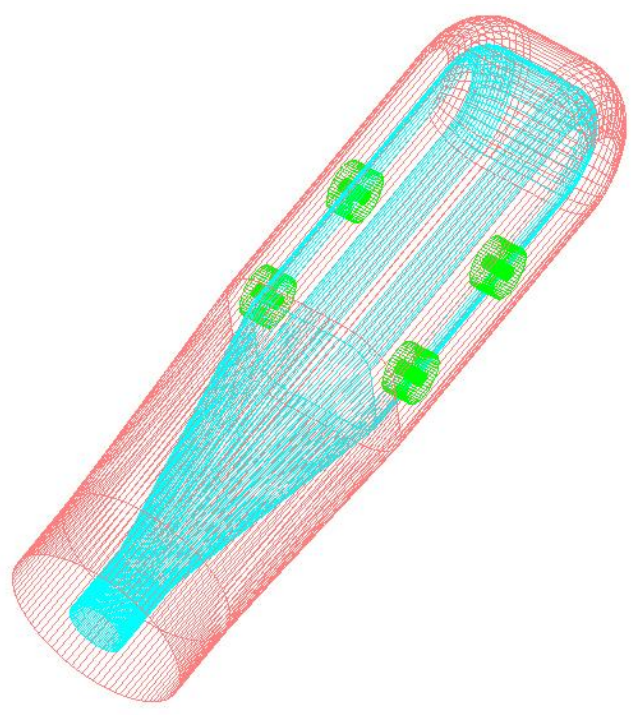

Fig. 19. Shorted phase shifter together with a matching taper.

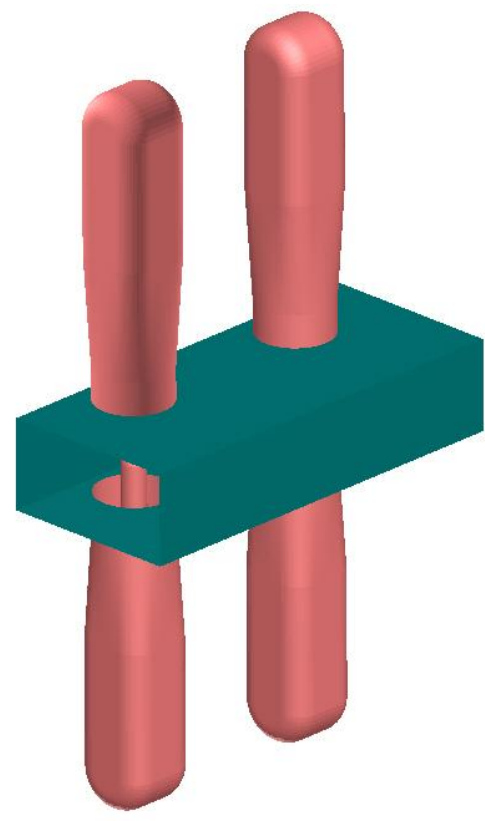

Fig. 20. Phase shifters connected in parallel in order to achieve high average power. 


\section{FAST FERROELECTRIC L-BAND TUNER FOR SUPERCONDUCTING CAVITIES}

\section{IIIc. Theoretical relation between phase shift and losses in a reflective phase shifter.}

For reference, a derivation is given here for the equations that govern the calculated results that are shown in the preceding sections. The ferroelectric ring in the line (Fig. 4) shorted from one side may be considered as a cavity strongly coupled to the line. In this case, the complex reflection coefficient $\Gamma$ is equal to

$$
\Gamma=\frac{\Gamma_{0}+1}{1+i Q_{l} x}-1=\frac{\Gamma_{0}-i Q_{l} x}{1+i Q_{l} x}=\frac{\left(\Gamma_{0}-\left(Q_{l} x\right)^{2}\right)-i Q_{l} x\left(1+\Gamma_{0}\right)}{1+\left(Q_{l} x\right)^{2}},
$$

where $\Gamma_{0}$ is reflection coefficient at the resonance. $Q_{l}$ is the cavity loaded quality factor, and $x$ is it's detuning, namely $x=\frac{\omega}{\omega_{0}}-\frac{\omega_{0}}{\omega} \approx \frac{2 \Delta f}{f}$. The line length may be chosen such that $\Gamma_{0}$ is real; but $\Gamma_{0} \sim 1$ because the coupling $\beta$ is strong, or $\beta \gg 1$. Note that

$$
\Gamma_{0}=\frac{\beta-1}{\beta+1} .
$$

The cavity is partially filled by dielectric with permittivity $\varepsilon$. If the dielectric constant is changed, the detuning of the cavity is equal to

$$
x=\frac{2 \Delta f}{f}=-\frac{\Delta \varepsilon}{2 W} \int_{V \varepsilon}|\vec{E}|^{2} d V
$$

where $\Delta \varepsilon$ is the dielectric constant change (in $\mathrm{F} / \mathrm{m}$ ), $W$ is the energy stored in the cavity, and $E$ is the electric field in the dielectric. Integral in Eq. 4 is calculated over the volume $V_{\varepsilon}$ occupied by dielectric. Introducing the new variables, $\eta=Q_{l} x$ and $\xi=\tan (\varphi)$, where $\varphi$ is the phase of the reflected signal, one can obtain from Eq. 1:

$$
\xi=\frac{\eta\left(1+\Gamma_{0}\right)}{\Gamma_{0}^{2}-\eta^{2}}
$$

From Eq. 5 one can determine the generalized cavity detuning $\eta$, namely

$$
\begin{array}{ll}
\eta=-\frac{1+\Gamma_{0}}{2 \xi}+\sqrt{\Gamma_{0}^{2}+\frac{\left(1+\Gamma_{0}\right)^{2}}{4 \xi}}, \quad \xi>0 ; \\
\eta=-\frac{1+\Gamma_{0}}{2 \xi}-\sqrt{\Gamma_{0}^{2}+\frac{\left(1+\Gamma_{0}\right)^{2}}{4 \xi}}, \quad \xi<0 ;
\end{array}
$$

For small $\xi$ one has 
FAST FERROELECTRIC L-BAND TUNER FOR SUPERCONDUCTING CAVITIES

$$
\eta \approx \frac{\Gamma_{0}^{2} \xi}{1+\Gamma_{0}}
$$

In order to change the phase of the reflected signal from $-\varphi_{0}$ to $-\varphi_{0}$ one should change the generalized cavity detuning by

$$
\Delta \eta=-\frac{1+\Gamma_{0}}{\xi_{0}}+\sqrt{4 \Gamma_{0}^{2}+\frac{\left(1+\Gamma_{0}\right)^{2}}{\xi_{0}}},
$$

or, for small $\varphi_{0}$

$$
\Delta \eta \approx \frac{2 \Gamma_{0}^{2} \xi_{0}}{1+\Gamma_{0}},
$$

where $\xi_{0}=\tan \left(\varphi_{0}\right)$. For strong coupling $\Gamma_{0}$ is very close to 1 , and the cavity detuning is

$$
\Delta \eta=-\frac{2}{\xi_{0}}+2 \sqrt{1+\frac{1}{\xi_{0}}}
$$

or, for small $\varphi_{0}$

$$
\Delta \eta \approx \xi_{0}
$$

On the other hand,

$$
Q_{l}=\frac{Q_{0}}{1+\beta}=\frac{W \omega}{P_{l o s s}(1+\beta)},
$$

and

$$
P_{l o s s}=\frac{4 \beta}{(1+\beta)^{2}} P_{0}
$$

where $P_{\text {loss }}$ are the power losses in the cavity and $P_{0}$ is the incident power. Combining Eq. 4,10 , 12 , and 13 , one has

$$
\frac{(1+\beta)}{\beta} \cdot \frac{\omega \Delta \varepsilon}{8 P_{0}} \int_{V \varepsilon}|\vec{E}|^{2} d V \approx \frac{\omega \Delta \varepsilon}{8 P_{0}} \int_{V \varepsilon}|\vec{E}|^{2} d V=-\frac{2}{\xi_{0}}+2 \sqrt{1+\frac{1}{\xi_{0}}} .
$$

From Eq. 14 one can fined the energy $W_{f}$ stored in the ferroelectric:

$$
W_{f}=\frac{\varepsilon}{2} \int_{V \varepsilon}|\vec{E}|^{2} d V=\frac{4 \varepsilon P_{0}}{\omega \Delta \varepsilon}\left(-\frac{2}{\xi_{0}}+2 \sqrt{1+\frac{1}{\xi_{0}}}\right) \approx \frac{4 \varepsilon P_{0}}{\omega \Delta \varepsilon} \phi_{0} .
$$

According to Eqs. 1 and 9,

$$
P_{\text {ferr }}=\operatorname{tg} \delta \cdot \omega W_{f},
$$




\section{FAST FERROELECTRIC L-BAND TUNER FOR SUPERCONDUCTING CAVITIES}

where $\delta$ is skin depth and $h$ is the ferroelectric ring thickness. Combining Eqs. 12-15 one obtains a simple expression for power losses:

$$
\frac{P_{\text {loss }}}{P_{0}} \approx 4 \operatorname{tg} \delta \frac{\phi_{0} \varepsilon}{\Delta \varepsilon} .
$$

This expression (Eq. 16) used above to calculate losses is seen to conform to intuitive expectations: device losses increase linearly as ferroelectric bulk losses increase, as phase shift increases, and inversely as tunability decreases. These features provide the theoretical basis that drives development of ferroelectric material towards lower losses and higher tunability.

\section{IIId. Experimental tests of ferroelectric assemblies}

During Phase I, Euclid TechLabs LLC supplied Omega-P with test assemblies that, for the first time, showed that brazing of ferroelectric ceramics to copper is feasible. Photographs of the test assemblies are in Figs. 21 and 22. These first test assemblies were supplied principally for DC breakdown tests, to gauge the efficacy of the brazing process. Earlier assemblies joined by epoxy glue did not prove as effective in withstanding voltages greater than about $5 \mathrm{kV}$, and in any case would not have been suitable for a high-power RF tuner that would need to endure high-temperature brazing and vacuum bakeout.

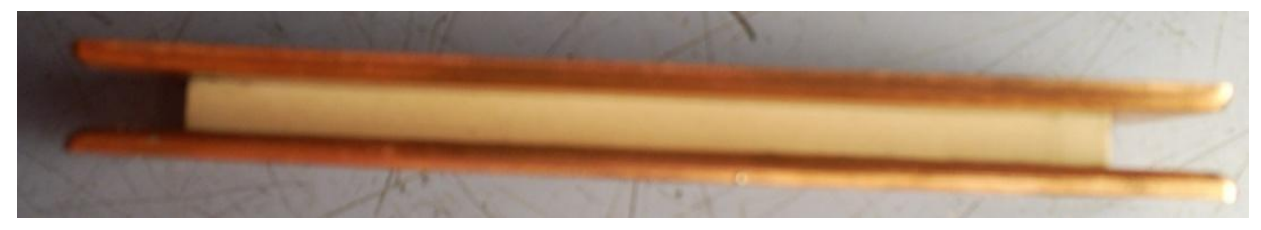

Fig. 21. One of the ferroelectric test assemblies built by Euclid TechLabs LLC and supplied to Omega-P for evaluation. Copper plates have rounded edges and insets to capture the brazed ferroelectric bar to avoid an exposed triple-point where DC breakdown could originate.

Fig. 23 shows a DC breakdown test underway, with the test stand under the polyethylene cover that allows $\mathrm{N}_{2}$ to be introduced to displace humid ambient air, the DC power supply on the right, and an oscilloscope for data accumulation at the left. Results are in Fig. 24, showing exposure times at top and number of breakdown events at bottom. 


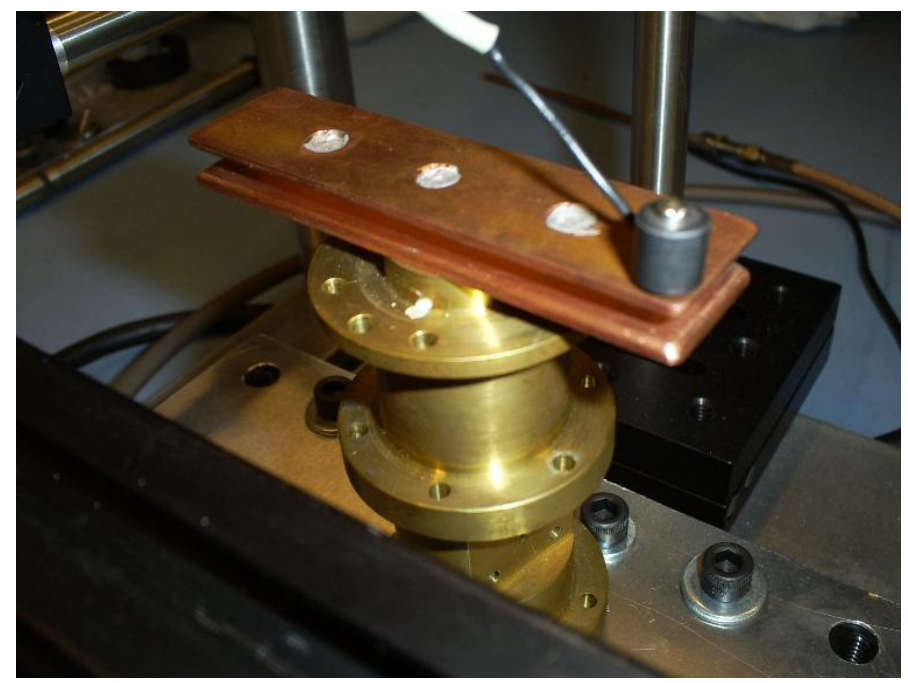

Fig. 22. Ferroelectric test assembly positioned on test stand for DC breakdown measurements. During application of up to $10 \mathrm{kV}$ the test stand is under a cover with air displaced by dry $\mathrm{N}_{2}$.

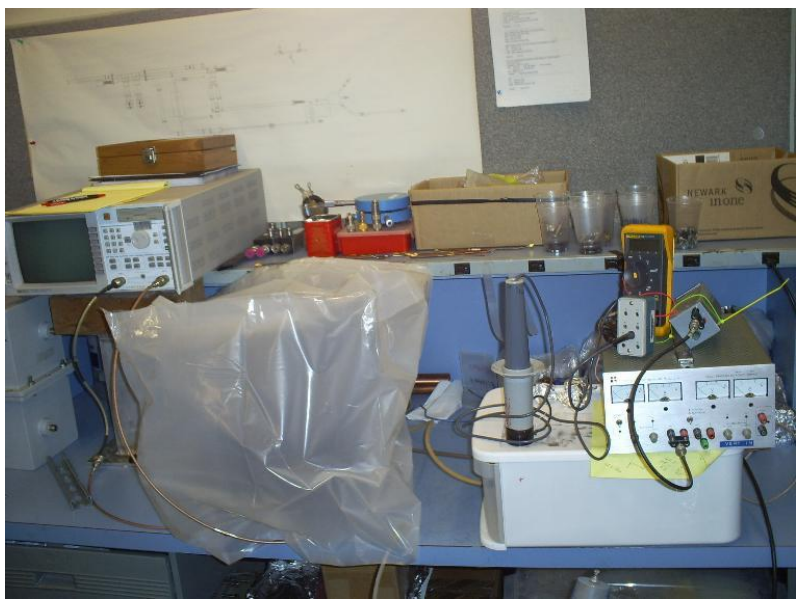

Fig. 23. Test setup for DC breakdown measurements on ferroelectric assemblies. 

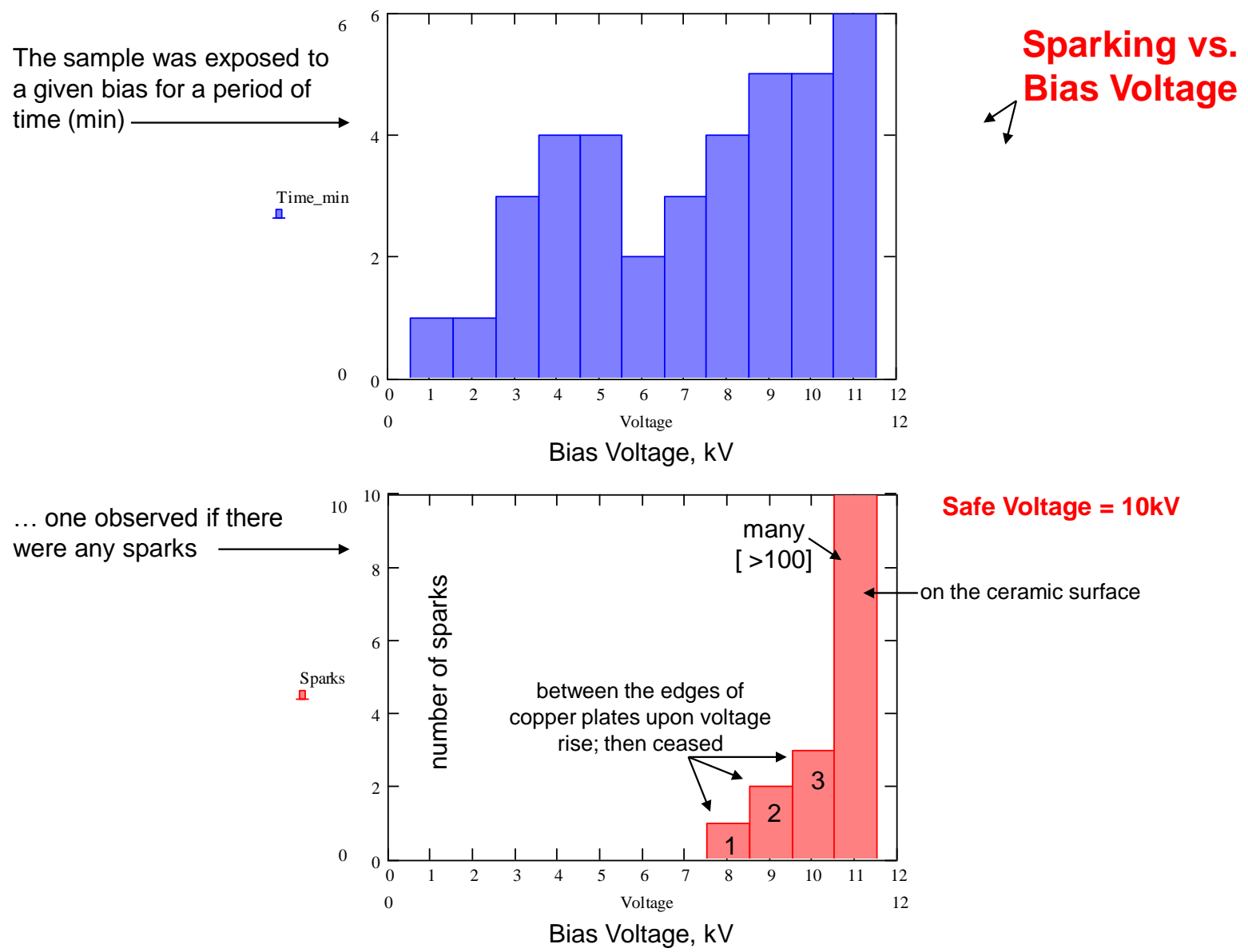

Fig. 24. Exposure times (top) and number of breakdown events (bottom), both as functions of applied voltage. After a few first breakdowns at the assembly edges, $10 \mathrm{kV}$ could be applied without breakdown. This corresponds to an electric field across the ferroelectric of $\sim 28 \mathrm{kV} / \mathrm{cm}$.

The observed breakdown data show that brazed ferroelectric assemblies supplied by Euclid TechLabs LLC can hold off voltages high enough to provide bias fields up to about 28 $\mathrm{kV} / \mathrm{cm}$. This value is expected to be sufficient as the ferroelectric material is further developed to lead to the $6 \%$ tunability that is sought.

Although not a principal goal of the Phase I project, preliminary low-power RF tests were carried out on the brazed (and glued) assemblies supplied by Euclid TechLabs LLC. These exercises were principally to refine the method of measurement, rather than to draw any conclusions as to the RF properties of this newly-formulated material while it is still in development by Euclid. Fig. 25 shows the assembly with RF loops in position for making a transmission measurement, results of which are displayed in Figs. 26 and 27. Observation of 


\section{Omega-P, Inc.}

\section{FAST FERROELECTRIC L-BAND TUNER FOR SUPERCONDUCTING CAVITIES}

clear discrete resonances shows the potency of this technique for future evaluation of Euclid assemblies to be built for the $704 \mathrm{MHz}$ tuner.
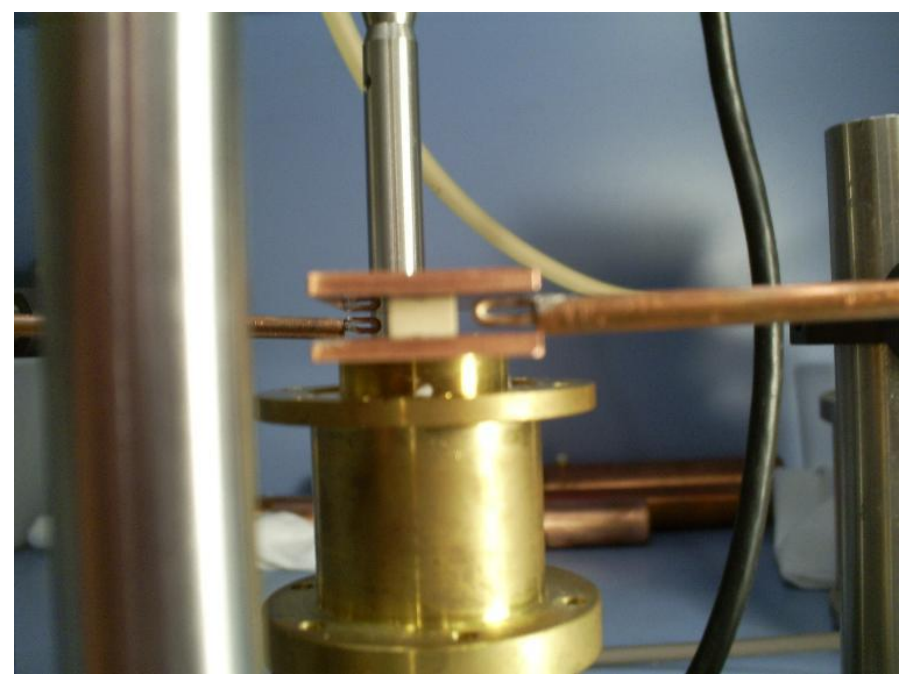

Fig. 25. Ferroelectric test assembly under test for RF transmission, with no HV bias applied.

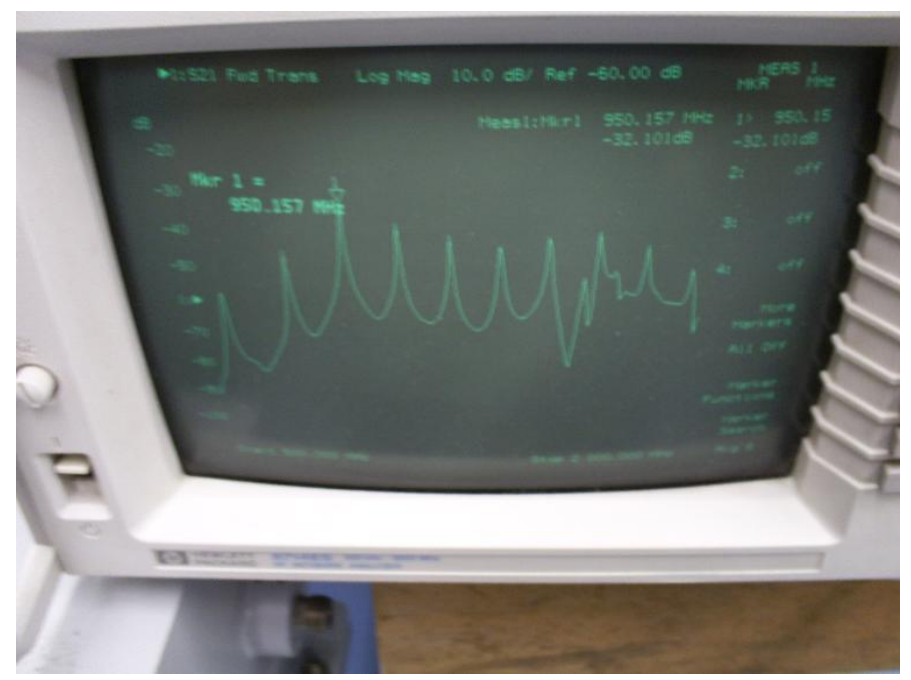

Fig. 26. Transmission spectrum as frequency is swept from 500 to $2000 \mathrm{MHz}$. Note the large number of prominent resonances. 


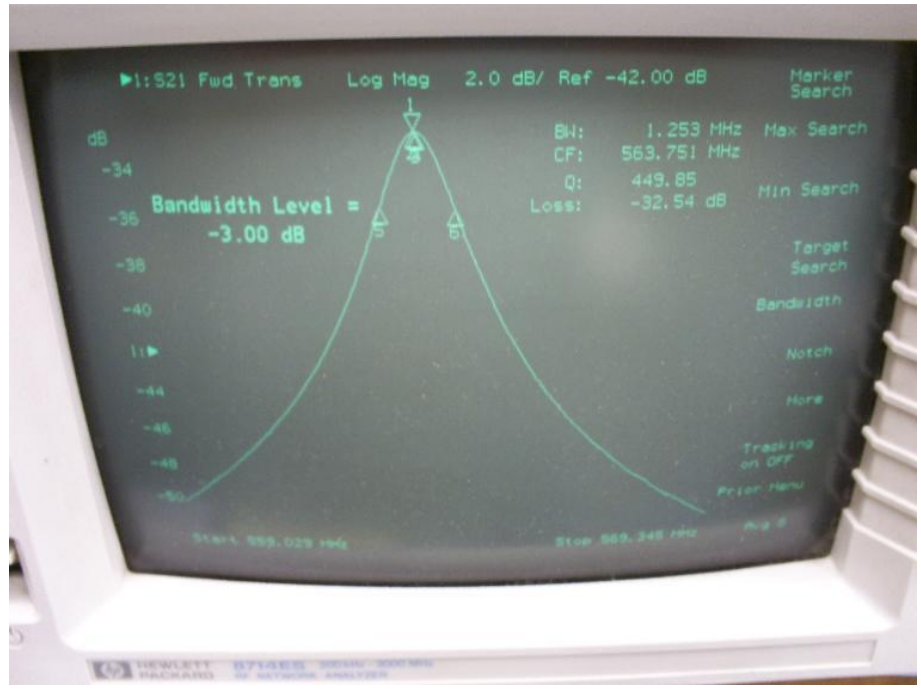

Fig. 27. Expanded view of RF transmission, showing a resonance at $563.75 \mathrm{MHz}$ with $Q=449$.

\section{IIIe. Power handling considerations}

High-power RF devices containing dielectrics have power handling limits due to bulk losses in the dielectrics, combined with relatively low thermal conductivities for dielectric materials. In this regard, ferroelectric materials are no exception. In this section of the proposal, power handling limits are estimated for the ferroelectric assemblies. Such limits are expected to be relatively severe, since the temperature rise of the ferroelectric must be small so as to not result in a change in dielectric constant with its associated change in phase for the tuner. For estimates here, a thermal conductivity value for the bulk ferroelectric material of $7.2 \mathrm{~W} / \mathrm{m}-{ }^{\circ} \mathrm{K}$ is assumed [A. Kanareykin, private communication]. Results presented here are for a donut-shaped ferroelectric element as shown in Fig. 7.

For a $1300 \mathrm{MHz}$ version, the element dimensions are height $h=10 \mathrm{~mm}$, diameter $2 R_{2}=$ $19.06 \mathrm{~mm}$, and the hole diameter $2 R_{1}=5 \mathrm{~mm}$. Fig. 27 shows the temperature rise in ${ }^{\circ} \mathrm{K}$ plotted along the altitude of the donut for an integrated average power dissipation of 1 Watt, assuming no temperature rise for the copper plates on top and bottom. From Fig. 18, it is seen that average loss about $6 \%$ for $100^{\circ}$ phase shift can be expected when the ferroelectric bulk loss tangent is $1 \times 10^{-3}$. Excluding losses in the copper parts gives a loss in the ferroelectric donut itself of 5\%. For the $1 \mathrm{~W}$ loss example in Fig. 28 with a peak temperature rise of $1.2{ }^{\circ} \mathrm{K}$, this implies a power handling capability for each ferroelectric assembly of $20 \mathrm{~W}$, and since the tuner 


\section{FAST FERROELECTRIC L-BAND TUNER FOR SUPERCONDUCTING CAVITIES}

would contain four assemblies in its simplest design, the device power handling capability would be $80 \mathrm{~W}$ for $\mathrm{a} \sim 1^{\circ} \mathrm{K}$ temperature rise. If a means to operate with a larger temperature rise were devised, such as providing external heating to "bias" the material well above room temperature, the power level could increase: a $10^{\circ} \mathrm{K}$ rise for $800 \mathrm{~W}$, a $20^{\circ} \mathrm{K}$ rise for $1.6 \mathrm{~kW}$, etc. Now if these results are scaled to $700 \mathrm{MHz}$, radii $R_{1}$ and $R_{2}$ for the ferroelectric donut will be increased by approximately a factor-of-two, but the height can be kept at $10 \mathrm{~mm}$. The ferroelectric loss tangent can be assumed to fall by a factor-of-two (if the material obeys the classical law $f \times Q=$ const.). Taken together, these factors result in a temperature rise that would be a factor-of-eight smaller than for the $1300 \mathrm{MHz}$ example, or for a power handling capability eight times higher, namely $6.4 \mathrm{~kW}$ for a $10^{\circ} \mathrm{K}$ temperature rise. One notes that this result $(6.4 \mathrm{~kW})$ is based on achieving a loss tangent of $5 \times 10^{-4}$ at $704 \mathrm{MHz}$ for the ferroelectric bulk material. If a higher power handling capability is required, remedies could include reduction in the loss tangent, operation with a range of acceptable temperature excursions exceeding $10^{\circ} \mathrm{K}$, and/or using a multiplicity of phase shifters in parallel, as shown in Fig. 20.

\section{Temperature along ferroelectric ring for $1 \mathrm{~W}$ loss/ring}

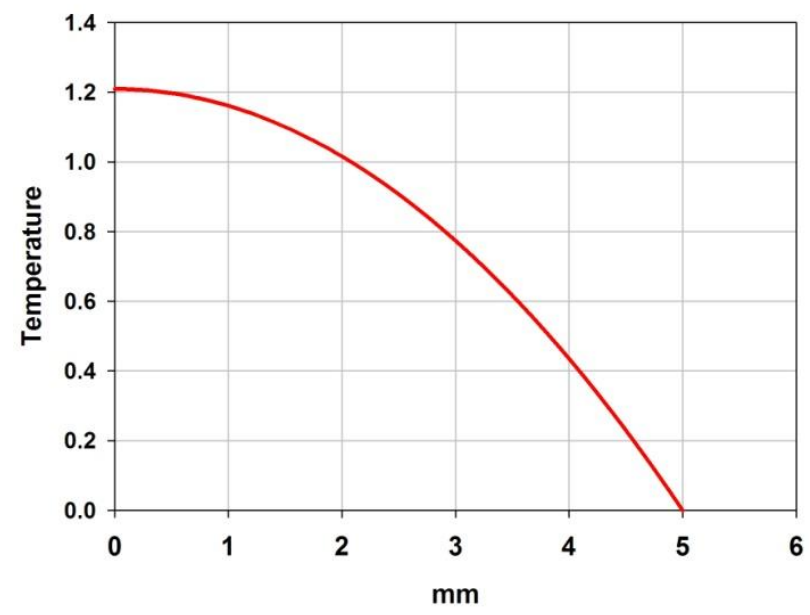

Fig. 28. Axial profile of temperature rise in a ferroelectric donut, for an absorbed power of 1 Watt, measured from the center of the donut out to the copper plates on top and bottom.

\section{CONCLUSIONS}

These results summarized above taken together lead to parameters for 1300 and $704 \mathrm{MHz}$ versions of the phase shifter that are summarized in Table I. Engineering design work that would have followed during Phase II would have been aimed towards building a $704 \mathrm{MHz}$ tuner prototype embodying two phase shifters with performance specifications as in the right-hand column of the Table. 
Omega-P, Inc.

Final Report on Phase I DoE SBIR grant DE-SC000 0922

FAST FERROELECTRIC L-BAND TUNER FOR SUPERCONDUCTING CAVITIES

Table I. Parameters of the phase shifter models.

\begin{tabular}{|c|c|c|}
\hline Parameter & & \\
\hline operating frequency, $\mathrm{MHz}$ & 1300 & 704 \\
\hline maximum pulse input power, $\mathrm{kW}$ & $500 *$ & $900 *$ \\
\hline number of ferroelectric assemblies & 4 & 4 \\
\hline maximum phase shift & $100^{\circ}$ & $100^{\circ}$ \\
\hline ferroelectric ring height, $\mathrm{mm}$ & 10 & 10 \\
\hline ferroelectric ring inner diameter, $\mathrm{mm}$ & 5 & 10 \\
\hline ferroelectric ring outer diameter, $\mathrm{mm}$ & 19.2 & 38.4 \\
\hline dielectric constant, zero bias & 300 & 300 \\
\hline dielectric constant, maximum bias & 280 & 280 \\
\hline phase shifter length, $\mathrm{mm}$ & 180 & 360 \\
\hline $\begin{array}{l}\text { maximum electric field in ferroelectric } \\
\mathrm{kV} / \mathrm{cm} \text { (at maximum pulse input power) }\end{array}$ & $12.5^{*}$ & $12.5^{*}$ \\
\hline power losses, dB & -0.26 & -0.13 \\
\hline temperature rise, ${ }^{\circ} \mathrm{K} / \mathrm{kW}$ (average) & 12 & 1.5 \\
\hline max. average power for $10^{\circ} \mathrm{K}$ heating, $\mathrm{kW}$ & 0.8 & 6.7 \\
\hline maximum bias electric field, $\mathrm{kV} / \mathrm{cm}$ & 15 & 15 \\
\hline maximum bias voltage, $\mathrm{kV}$ & 15 & 15 \\
\hline
\end{tabular}

*The maximum peak power capability of the phase shifters will be governed by rf breakdown limits, which will be set by engineering details for the actual design chosen. The 500 and 900 $\mathrm{kW}$ figures given here is merely illustrative. Since it is the average power that determines temperature rise and change in dielectric constant for the ferroelectric material, the actual peak power limit will follow from the acceptable temperature rise and the duty factor.

A summary of results under this grant follows, with reference to the originally proposed tasks (see Section 0, p. 2).

Task a: Further optimize the design for a full-scale triple-decker $700 \mathrm{MHz}$ tuner.

Discussion of issues with the triple-decker planar tuner design are provided in Section IIIa; these are summarized as follows: Experience with the triple-decker concept demonstrated problems with high-voltage breakdown and brazing of ceramics to copper walls. In addition, the concept required two types of ceramic elements: ferroelectric bars and linear ceramic matching bars. The radial line reflecting phase shifter requires a ferroelectric ring of substantial physical size that appeared to be difficult to sinter, metalize and braze. Thus, after detailed analysis of electrodynamic and technological problems of both concepts, these were downgraded in priority in favor of a new concept based on use of a number of small ferroelectric ring assemblies. These assemblies can be standardized, and are of 


\section{FAST FERROELECTRIC L-BAND TUNER FOR SUPERCONDUCTING CAVITIES}

relatively small size that should be relatively easy to manufacture, individually tuned, cooled, and tested. Furthermore, this new phase shifter design requires no linear ceramic matching elements.

Task b: Carry out simulation studies of the radial line reflecting tuner, with the aim of comparing its performance with the planar triple-decker design so as to select the superior design for full development during Phase II.

Discussion of unappealing issues with the radial line reflecting tuner-similar to those with the triple-decker planar design - are given in Section IIIb. This design was also abandoned, in favor of a new version developed during Phase I that incorporated donut-shaped ferroelectric resonant cavity elements in a transmission or reflection microwave circuit. This configuration appears to constitute a breakthrough design with superior electrical, thermal, and mechanical properties, as compared with either the planar triple-decker concept, or the radial line concept. Details of the tuner using donut-shaped ferroelectric cavities are given in Section IIIb. Parameters for 1300 and $704 \mathrm{MHz}$ versions of this tuner are in Table I.

Task c: If results of Task b are sufficiently encouraging, build a $1300 \mathrm{MHz}$ prototype for lowpower cold tests, and carry out the tests to confirm the predictions.

A full-scale prototype design was not built during Phase I, since issues arose from the need for a reliable technique for brazing ferroelectric ceramics to the surrounding metallic structure. So planar geometry test structures were built and tested to evaluate performance of planar bars of ferroelectric ceramic as supplied by Euclid Techlabs LLC. Results of these measurements are given in Section IIId.

Task d: Coordinate with Euclid Techlabs LLC to improve the recipe for ferroelectric material with the aim of lowering its loss tangent without undue sacrifice in tunability, and to develop a technique for reliable low-temperature soldering or brazing to copper plates of metalized ferroelectric and ceramic bars. Attention will be directed towards developing a technique for the braze fillet to cover triple-point corners, so as to guard against field-enhanced breakdown at those joints. Test both improvements at $1300 \mathrm{MHz}$ in one-third scaled three-deck structure, or in radial line tuner.

Euclid Techlabs LLC did deliver to Omega-P a variety of ferroelectric and linear ceramic bars with and without metallic coatings, for tests on soldering these to surrounding planar metallic structures. Coordinated efforts with Euclid were undertaken to optimize the metallic coating of the ferroelectric and ceramic bars, while improving the ferroelectric properties such as dielectric constant, loss tangent, tenability, and reduction of tuning hysteresis.

Task $\boldsymbol{e}$ : Develop or acquire an improved fast high-voltage pulser and/or pulser circuit for rapid phase shifting. Test one or both tuner at low power to determine their shortest response time. 


\section{Omega-P, Inc.}

Final Report on Phase I DoE SBIR grant DE-SC000 0922

\section{FAST FERROELECTRIC L-BAND TUNER FOR SUPERCONDUCTING CAVITIES}

During Phase I, it was decided that when need for a pulsed power supply emerged, Omega-P's existing pulser used for firing plasma and electron-beam switches would be more than adequate to satisfy needs for anticipated testing. Thus further effort to satisfy this task was not needed.

Task f: Assemble Phase I results and confer with collaborators to plan and compose a Phase II project and proposal.

This task was completed in full.

Task g: Write a final report, and as work progresses deliver oral presentations at relevant conferences and workshops, and at sponsor's reviews as requested. Publish significant results in refereed journals.

This task was completed in full. The relevant publication in a refereed journal is "Fast ferroelectric phase shifters for energy recovery linacs", S. Yu Kazakov, S. V. Shchelkunov, V. P. Yakovlev, A. Kanareykin, E. Nenasheva, J. L. Hirshfield, Phys. Rev. ST Accel. Beams 13, 11350 (2010). 
Omega-P, Inc.

Final Report on Phase I DoE SBIR grant DE-SC000 0922

FAST FERROELECTRIC L-BAND TUNER FOR SUPERCONDUCTING CAVITIES

\section{REFERENCES}

1. S. Simrock., G. Petrosyan, A. Facco, V. Zvyagintsev, S. Andreoli, and R. Paparella, "First Demonstration of Microphonic Control of a Superconducting Cavity with a Fast Piezoelectric Tuner", PAC2003, Portland, May 11-16, 2003, p.470.

2. M. Liepe and S. Belomestnykh, "Microphonics Detuning in the $500 \mathrm{MHz}$ Supercondicting CESR Cavities," PAC2003, Portland, May 11-16, 2003, pp.1326-1328.

3. Y. Kang, M. Tiefenback, and P. Chevtsov, "Reactive RF Tuning for Compensation of a Detuned Accelerating Cavity," Linac 2002, Gyeongju, Korea, 2002, pp.733-735.

4. $\quad$ Martin Dohlus, DESY, Feb. 2004.

5. T. Zwart, D. Cheever, W.S. Graves, D. Wang, A. Zolfaghari, "Optimal RF System For Lightly Loaded Superconducting Structures”, Proceedings of the 2004 FEL Conference, pp. 542-545; see also web.elettra.trieste.it/fel2004/proceedings/papers/TUPOS57/TUPOS57.PDF

6. D. Valuch, H. Frischholz, J. Tückmantel, and C. Weil, "First Results With A Fast Phase And Amplitude Modulator For High Power RF Applications," EPAC2004, pp. 959-961.

7. D. Horan, and E. Cherbak, "Fast-Ferrite Tuner Operation On A 352-MHz Single-Cell RF Cavity At The Advanced Photon Source," PAC2003, pp. 1177-1179.

8. B. Foster, I. Gonin, T. Khabiboulline, A. Makarov, N. Solyak, I. Terechkine, and D. Wildman, "High Power Phase Shifter,” PAC2005, PAC2005, Knoxville, May 16-20, 2005, p. 3123-3125.

9. M. Liepe and S. Belomestnykh,'RF Parameters and Field Stability Requirements for the Cornell ERL Prototype", PAC2003, Portland, May 11-16, 2003, pp.1326-1328.

10. I. Ben-Zvi, "The ERL High-Energy Cooler for RHIC”, EPAC2006, Edinburgh, 2006, pp. 940-944. See also R. Calaga, "SRF Cavities for High Current ERLs," BNL, January 23, 2006, http://www.bnl.gov/cad/ecooling/docs/PDF/MAC_01_06/calaga.pdf

11. V.P. Yakovlev, S.Yu. Kazakov, and J.L. Hirshfield, "1.3 GHz Electrically-Controlled Fast Ferroelectric Tuner,” EPAC2006, Edinburgh, June 26-30, 2006, pp. 487-489. 